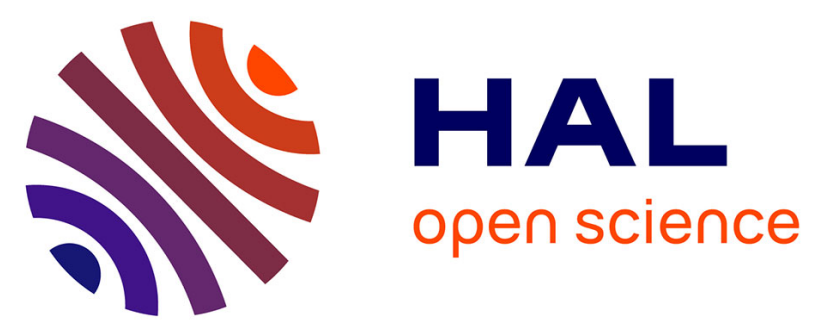

\title{
On the use of large time steps with an energy momentum conserving algorithm for non-linear hypoelastic constitutive models
}

\author{
Ludovic Noels, Laurent Stainier, Jean-Philippe Ponthot
}

\section{To cite this version:}

Ludovic Noels, Laurent Stainier, Jean-Philippe Ponthot. On the use of large time steps with an energy momentum conserving algorithm for non-linear hypoelastic constitutive models. International Journal of Solids and Structures, 2004, 41 (3-4), pp.663-693. 10.1016/j.ijsolstr.2003.09.032 • hal-01007159

\author{
HAL Id: hal-01007159 \\ https://hal.science/hal-01007159
}

Submitted on 10 Nov 2021

HAL is a multi-disciplinary open access archive for the deposit and dissemination of scientific research documents, whether they are published or not. The documents may come from teaching and research institutions in France or abroad, or from public or private research centers.
L'archive ouverte pluridisciplinaire HAL, est destinée au dépôt et à la diffusion de documents scientifiques de niveau recherche, publiés ou non, émanant des établissements d'enseignement et de recherche français ou étrangers, des laboratoires publics ou privés.

\section{(ㄷ)(1) $\$$}

Distributed under a Creative Commons Attribution - NonCommerciall 4.0 International 


\title{
On the use of large time steps with an energy momentum conserving algorithm for non-linear hypoelastic constitutive models
}

\author{
L. Noels, L. Stainier, J.-P. Ponthot * \\ Department de Thermomee' canique-LTAS, University of Li`eege, 1 Chemin des Chevreuils, B-4000 Li`eege, Belgium
}

This paper presents an extension of the energy momentum conserving algorithm, developed by the authors for hypoelastic constitutive models. For such a material, contrarily to hyperelastic models, no potential can be defined, and thus the conservation of the energy is ensured only if the elastic work of deformation can be restored by the scheme. In a previous paper, we proposed a new expression of internal forces at the finite element level which is shown to verify this property. We also demonstrated that the work of plastic deformation is positive and consistent with the material model. In this paper, the second order terms that were neglected in the previous work are now taken into account. Several numerical applications are presented to demonstrate the necessity of taking these terms into account once large time step sizes are used. The limitations of the Newmark algorithm in the non-linear range are also illustrated.

Keywords: Energy-momentum conserving; Dynamics; Hypoelastic constitutive model; Large strain plasticity; Finite elements

\section{Introduction}

One can resort to two families of algorithms to integrate the equations of evolution of dynamical systems: the implicit family and the explicit family. In this paper, we focus on the implicit family. The most widely used implicit algorithm is the Newmark (1959) algorithm (see also Belytschko and Hughes, 1983; Hughes, 1987; Belytschko et al., 2000). For linear models, this algorithm is unconditionally stable if some conditions on the parameters are verified. For non-linear models, Belytschko and Schoeberle (1975) and Hughes (1977) proved that the discrete energy is bounded if it remains positive. Nevertheless, Hughes et al. (1978), Simo et al. (1992) and Kane et al. (2000) have proved that, in the non-linear range, the Newmark algorithm remains stable only for small time step sizes. Moreover, for a step between times $t_{n}$ and $t_{n+1}$, the angular momentum is conserved between the times $t_{n-\frac{1}{2}}$ and $t_{n+\frac{1}{2}}$ but not between the times of computation $t_{n}$

\footnotetext{
* Corresponding author. Tel.: +32-4-3669310; fax: +32-4-3669141.

E-mail address: jp.ponthot@ulg.ac.be (J.-P. Ponthot).
} 
and $t_{n+1}$ (Simo et al., 1992). To avoid divergence due to the numerical instabilities, numerical damping was thus introduced, leading to the generalized- $\alpha$ methods (Belytschko and Hughes, 1983; Hughes, 1987; Belytschko et al., 2000; Chung and Hulbert, 1993; Géradin and Cardona, 2000). Nevertheless, the unconditional stability of these methods can be proved only for linear systems. Another method is to set the Newmark parameters so as to dissipate energy (Ponthot, 1995). But these techniques have the disadvantage to also damp the physical modes, leading to a lack of accuracy. Therefore a new kind of dynamics integration algorithms has appeared that verify the mechanical laws of conservation (i.e. conservation of linear momentum, angular momentum and total energy) and that remain stable in the non-linear range.

To demonstrate the stability, these new algorithms were not studied on a linear system as the previous ones, but on the non-linear elements under considerations. The first algorithm verifying these properties was described by Simo and Tarnow (1992) and Simo and Gonzalez (1994). They called this algorithm Energy Momentum Conserving Algorithms or EMCA. It consists in a mid-point scheme with an adequate evaluation of the internal forces. This adequate evaluation was given for a Saint Venant-Kirchhoff hyperelastic material. This scheme was further extended to shells by Simo and Tarnow (1994), Zhong and Crisfield (1998), Kuhl and Crisfield (1999) and Sansour et al. (2002), and was extended to mixed finite shells by Miehe and Schröder (2001). It was also extended to rods by Romero and Armero (2002b), to composite laminates by Brank (2002) and to multi-body dynamics by Géradin and Cardona (2000) and Briseghella et al. (1999). A generalization to other hyperelastic models was given by Laursen and Meng (2001), who iteratively solve a new equation for each Gauss point to determine the adequate second Piola-Kirchhoff stress tensor. Another solution that avoids this iterative procedure leads to a general formulation of the second Piola-Kirchhoff stress tensor, as given by Gonzalez and Simo (1996) and Gonzalez (2000). This formulation is valid for general hyperelastic materials. The EMCA was recently extended to dynamic finite deformation plasticity by Meng and Laursen (2001, 2002). In such a formulation, the algorithm remains energy conserving when no plastic deformation occurs, and "dissipates energy in a manner consistent with the physical model in use" (sic) when plastic deformation occurs.

In the same context, for contact treatment, a penalty method was developed to simulate non-frictional and frictional contact interactions by Armero and Petöcz $(1998,1999)$. This method allows surface penetration but ensures conservation of the energy for frictionless problems and consistent dissipation for frictional ones. Laursen and Chawla (1997), Chawla and Laursen (1998) and Laursen (2002) developed an alternative method to simulate non-frictional and frictional contact. This method, that also allows surface penetration, is unconditionally dissipative (even for the frictionless problems) for the penalty and augmented Lagrangian method. The unconditional dissipation allows the method to be stable. More recently, Laursen and Love (2002) extended these methods for frictionless contact, with the use of a contact velocity correction that imposes the geometric admissibility and leads to a conserving algorithm. The use of a contact potential was also proposed by Goicolea and Garcia Orden (2000) to simulate the frictional contact. Finally, these contact methods were used in the context of quasi-rigid bodies (small deformations added to a rigid transformation), such as gear trains, by Demkowicz and Bajer (2001) and Bajer and Demkowicz (2002).

Independently of contact, to avoid the lack of convergence due to the presence of high frequency modes, numerical dissipation was introduced in the conserving algorithms (hyperelastic materials) by Armero and Romero (1999, 2001a,b) and Romero and Armero (2000, 2002a). This algorithm preserves the angular momentum, contrarily to the generalized $\alpha$ algorithms and is called Energy Dissipative Momentum Conserving algorithm or EDMC. This scheme could be second order accurate by the introduction of new relations, leading to twice more equations. This EDMC method was extended to beams by Ibrahimbegovic and Mamouri (2002). Another solution to verify all conservation equations is to use the generalized- $\alpha$ method or the EDMC algorithm, but to augment these algorithms with energy and momentum constraints (Hughes et al., 1978; Kuhl and Crisfield, 1999; Kuhl and Ramm, 1996, 1999). This solution is called either Constraint Energy Momentum Algorithm (CEMA) in the first case or Modified Energy-Momentum Method (MEMM) in the second case. In such an augmented method, the dissipated energy of the high frequency modes is added 
to the energy of the low frequency modes. Let us note that in these works (Kuhl and Crisfield, 1999; Kuhl and Ramm, 1996, 1999), the velocity dissipation introduced later by Armero and Romero (1999, 2001a,b) and Romero and Armero (2000, 2002a) to dissipate the high frequency is not present.

Finally, the properties of conservation can be reached, as proposed by Betsch and Steinmann (2000a,b, 2001), by using a Petrov-Galerkin finite element method. Graham et al. (2002) have proved that this method is equivalent to the Simo and Gonzalez (1994) one, if some conditions are respected. Bauchau and Theron (1996) and Bottasso and Borri (1997) developed a stable conserving (Energy Preserving) and dissipative (Energy Decaying) method, for the simulation of non-linear beams, by the use of an approximation of, respectively, the time Galerkin method and the time discontinuous Galerkin method. This approximation consists in the transformation of the Galerkin time integral into a finite difference. These algorithms (Preserving and Decaying) were extended to non-linear elasto-dynamics by Bauchau and Joo (1999). Let us note that the Preserving scheme is second order accurate and is similar to Simo and Tarnow (1992). The Decaying scheme, is third order accurate but requires to solve twice more equations and cannot be modified to control the dissipation. Another Energy Preserving/Decaying algorithm can be obtained with a RungeKutta method. This method was used to compute non-linear beams by Bottasso and Borri (1998) and to compute multi-body dynamics by Borri et al. (2001) and Bottasso et al. (2001). These two methods (first one based on Galerkin method, and second one based on a Runge-Kutta method) were unified by Bauchau and Bottasso (1999) for multi-body dynamics. This unification leads to the use of finite difference approximation of the Galerkin method (Energy Preserving) or of the time discontinuous Galerkin method (Energy Decaying) to simulate shells, cables and beams dynamics (Bauchau et al., 2002, 2003; Bottasso et al., 2002). In this case, the Energy Decaying scheme is third order accurate (but the number of equations to be solved is multiplied by 2) and the numerical dissipation can be controlled.

All the conserving methods described above were established for hyperelastic materials. In a previous work (Noels et al., 2004), we have developed a new expression of the internal forces, ensuring the conservation laws of the mechanics for a hypoelastic constitutive model. But, in this formulation, assuming small time step size, we have neglected the second order term on the increment of the plastic strain. In this paper, we prove that with the use of large time steps (according to the plastic deformation rate), those terms are necessary to obtain an accurate and a stable solution. In Section 2, the methodology for the evaluation of the stress tensor in hypoelastic materials and its spatial integration is recalled. In Section 3 we show how to compute the internal forces to verify the conserving relations for a hypoelastic material using the final rotation scheme. Moreover, we prove that this adaptation remains consistent when plastic deformation occurs. Finally, in Section 4, numerical examples illustrate the advantages and the disadvantages of the conserving algorithm, considering or not the second order terms.

\section{The hypoelastic material model}

First, the notations used in this paper are detailed. Next, the method used for computation of the stress tensor in hypoelastic materials is explained. The plastic deformations are taken into account. Finally, the spatial integration of this stress tensor to obtain the internal forces is established in a finite element framework.

\subsection{Preliminaries}

Let the configuration $n$ be the configuration computed after $n$ time steps (i.e. at time $t_{n}$ ). Let $\vec{x}^{n}$ be the deformation mapping (coordinates) in the configuration $n$, and let $\left[\vec{x}^{n}\right]^{\xi}$ be the coordinates of the position for node $\xi\left(\xi \in[1, N]\right.$ with $N$ the number of nodes of the element) in the configuration $n$. With $\varphi^{\xi}$ the shape function evaluated related to node $\xi$, it comes (Einstein's notations are used) 


$$
\begin{aligned}
& \vec{x}=\varphi^{\xi} \vec{x}^{\xi} \\
& \dot{\vec{x}}=\varphi^{\xi} \dot{\vec{x}}^{\xi} \\
& \ddot{\vec{x}}=\varphi^{\xi} \ddot{\vec{x}}^{\xi}
\end{aligned}
$$

The gradient of deformation (two point tensor) $\mathbf{F}$ between configurations $m$ and $n$ is indicated by $\mathbf{F}_{m}^{n}$. This tensor is defined by

$$
\mathbf{F}_{m}^{n}=\frac{\partial \vec{x}^{n}}{\partial \vec{x}^{m}}
$$

Tensor $\mathbf{f}$ represents $\mathbf{F}^{-1}$. When $m$ refers to the initial configuration, the gradient of deformation is written as

$$
\mathbf{F}_{0}^{n}=\frac{\partial \vec{x}^{n}}{\partial \vec{x}^{0}}
$$

with

$$
\mathbf{F}_{0}^{n}=\mathbf{F}_{m}^{n} \mathbf{F}_{0}^{m}
$$

According to the theorem of polar decomposition, this gradient tensor can be decomposed into a rotation tensor $\mathbf{R}$ and a symmetric positive definite deformation tensor $\mathbf{U}$ ( $\mathbf{I}$ is the identity tensor)

$$
\begin{aligned}
& \mathbf{F}_{m}^{n}=\mathbf{R}_{m}^{n} \mathbf{U}_{m}^{n} \\
& \mathbf{U}_{m}^{n}=\mathbf{U}_{m}^{n^{\mathrm{T}}} \\
& \mathbf{R}_{m}^{n^{\mathrm{T}}} \mathbf{R}_{m}^{n}=\mathbf{I}
\end{aligned}
$$

The determinant of $\mathbf{F}_{m}^{n}$ is denoted by scalar $J_{m}^{n}$. The relation between density $\rho$ of the body and this determinant is

$$
\rho^{n}=\frac{\rho^{0}}{J_{0}^{n}}
$$

Green-Lagrange strain tensor $\mathbf{G L}_{m}^{n}$ is defined as

$$
\mathbf{G L}_{m}^{n}=\frac{1}{2}\left[\mathbf{F}_{m}^{n^{\mathrm{T}}} \mathbf{F}_{m}^{n}-\mathbf{I}\right]=\frac{1}{2}\left[\mathbf{U}_{m}^{n} \mathbf{U}_{m}^{n}-\mathbf{I}\right]
$$

and Almansi strain tensor $\mathbf{A}_{m}^{n}$ is defined as

$$
\mathbf{A}_{m}^{n}=\frac{1}{2}\left[\mathbf{I}-\mathbf{f}_{m}^{\mathbf{n}^{\mathrm{T}}} \mathbf{f}_{m}^{n}\right]=\mathbf{f}_{m}^{\mathrm{n}^{\mathrm{T}}} \mathbf{G} \mathbf{L}_{m}^{n} \mathbf{f}_{m}^{n}=\frac{1}{2} \mathbf{R}_{m}^{n}\left[\mathbf{I}-\mathbf{U}_{m}^{n^{-1}} \mathbf{U}_{m}^{n^{-1}}\right] \mathbf{R}_{m}^{n^{\mathrm{T}}}
$$

Natural strain tensor $\mathbf{E}_{m}^{n}$ is also computed from $\mathbf{F}$ or might be computed from $\mathbf{G L}$ or from $\mathbf{A}$

$$
\mathbf{E}_{m}^{n}=\frac{1}{2} \ln \left[\mathbf{F}_{m}^{n^{\mathrm{T}}} \mathbf{F}_{m}^{n}\right]=\frac{1}{2} \ln \left[2 \mathbf{G L}_{m}^{n}+\mathbf{I}\right]=-\frac{1}{2} \ln \left[\mathbf{I}-2 \mathbf{R}_{m}^{n^{\mathrm{T}}} \mathbf{A}_{m}^{n} \mathbf{R}_{m}^{n}\right]
$$

The Cauchy stress tensor is evaluated in the configuration $n$ and is referred to as $\Sigma^{n}$. If the internal forces are pushed backward into the initial configuration, the stress tensor used is the second Piola-Kirchhoff tensor (S), that is evaluated with respect to configuration $n$, as

$$
\mathbf{S}^{n}=J_{0}^{n} \mathbf{f}_{0}^{n} \Sigma^{n} \mathbf{f}_{0}^{n^{\mathrm{T}}}
$$

\subsection{Stress tensor computation}

By definition, for a hyperelastic material, there exists a potential $\phi(\mathbf{G L})$ from which the second PiolaKirchhoff stress tensor is computed 


$$
\mathbf{S}^{n}=\rho^{0} \frac{\partial \phi\left(\mathbf{G L}_{0}^{n}\right)}{\partial \mathbf{G L}_{0}^{n}}
$$

For hypoelastic constitutive laws, the Cauchy stress tensor is computed from a stress increment $\Delta \boldsymbol{\Sigma}_{n}^{n+1}$ between two successive configurations. The final rotation scheme (Nagtegaal, 1982; Nagtegaal and Veldpaus, 1982, 1984; Ponthot, 1995, 2002) is defined by the following relation:

$$
\Sigma^{n+1}=\mathbf{R}_{n}^{n+1}\left[\Sigma^{n}+\Delta \Sigma_{n}^{n+1}\right] \mathbf{R}_{n}^{n+1^{\mathrm{T}}}
$$

Let us define, with an exponent $c$, the corotational value that is the term before the final rotation scheme. For example, the corotational stress is defined by

$$
\Sigma^{c^{n+1}}=\Sigma^{n}+\Delta \Sigma_{n}^{n+1}
$$

This scheme presents some important properties:

- It is incrementally objective (i.e. the stress tensor is exactly updated for a rigid body motion).

- No parasitic volume variation is generated (i.e. the scheme does not lead to a variation of the volume for a rigid motion).

If the material behavior is elastic, the stress increment is deduced from the natural strain tensor

$$
\Delta \Sigma_{n}^{n+1}=\mathscr{H}: \mathbf{E}_{n}^{n+1}
$$

with $\mathscr{H}$ denoting the Hooke fourth order tensor ( $k$ is the bulk modulus and $g$ the shear modulus)

$$
\mathscr{H}_{i j k l}=k \delta_{i j} \delta_{k l}+g\left[\delta_{i k} \delta_{j l}+\delta_{i l} \delta_{j k}-\frac{2}{3} \delta_{i j} \delta_{k l}\right]
$$

and operation $\mathscr{H}: \mathbf{E}$ is defined by $\mathscr{H}_{i j k l} \mathbf{E}_{k l}$. For an elastoplastic or elastoviscoplastic material, relation (12) and relation (14) can only be directly used when the material remains elastic. If plastic deformations occur, the relation (12) becomes

$$
\Sigma^{\mathbf{c}^{n+1}}=\left[\Sigma^{n}+\Delta \Sigma_{n}^{n+1}-\mathbf{s}^{\mathbf{c}}\right]
$$

where $\mathbf{s}^{\mathbf{c}}$ is the purely deviatoric correction tensor resulting from the radial return mapping ( $J 2$ plasticity) (Wilkins, 1964; Maenchen and Sack, 1964; Simo and Hughes, 1998; Ponthot, 2002). It is evaluated by the following method: the elastic predictor $\mathbf{s}^{\mathrm{e}}$ is defined by the deviatoric part of $\Sigma^{n}+\Delta \Sigma_{n}^{n+1}$ where $\Delta \Sigma_{n}^{n+1}$ is given by (14). If $\alpha$ defines the heredity backstress tensor, corotational normal tensor $\mathbf{N}^{\mathrm{c}}$ is defined by

$$
\mathbf{N}^{\mathbf{c}}=\frac{\mathbf{s}^{\mathbf{e}}-\boldsymbol{\alpha}}{\sqrt{\left[\mathbf{s}^{\mathbf{e}}-\alpha\right]:\left[\mathbf{s}^{\mathbf{e}}-\alpha\right]}}
$$

where operation $\mathbf{a}: \mathbf{b}$ is defined by $\mathbf{a}_{i j} \mathbf{b}_{i j}$. If scalar $\varepsilon^{\mathrm{p}}$ defines the equivalent plastic strain, if scalar $\Sigma_{\mathrm{v}}$, function of $\varepsilon^{\mathrm{p}}$, defines the subsequent von Mises yield stress, and if $\bar{\alpha}$, function of $\varepsilon^{\mathrm{p}}$, defines the equivalent heredity, then scalar $\gamma$ can be defined such as to have (Ponthot, 1995, 2002)

$$
\begin{aligned}
& \varepsilon^{\mathrm{p}^{n+1}}=\varepsilon^{\mathrm{p}^{n}}+\sqrt{\frac{2}{3}} \gamma \\
& \sum_{\mathrm{v}}^{n+1}\left(\varepsilon^{\mathrm{p}^{n+1}}\right)=\sum_{\mathrm{v}}^{n+1}(\gamma) \\
& \boldsymbol{\alpha}^{\mathbf{c}^{n+1}}=\boldsymbol{\alpha}^{n}+\sqrt{\frac{2}{3}}\left[\bar{\alpha}\left(\varepsilon^{\mathrm{p}^{n+1}}\right)-\bar{\alpha}\left(\varepsilon^{\mathrm{p}^{n}}\right)\right] \mathbf{N}^{\mathbf{c}} \\
& \mathbf{s}^{\mathbf{c}}=2 g \gamma \mathbf{N}^{\mathbf{c}}
\end{aligned}
$$

where the scalar value of $\gamma$ is solved from the von Mises criterion (Ponthot, 2002) evaluated at time $t_{n+1}$, 


$$
\left[\mathbf{s}^{\mathbf{e}}-2 g \gamma \mathbf{N}^{\mathbf{c}}-\boldsymbol{\alpha}^{\mathbf{c}^{n+1}}(\gamma)\right]:\left[\mathbf{s}^{\mathbf{e}}-2 g \gamma \mathbf{N}^{\mathbf{c}}-\boldsymbol{\alpha}^{\mathbf{c}^{n+1}}(\gamma)\right]=\frac{2}{3}\left[\sum_{\mathrm{v}}^{n+1}(\gamma)\right]^{2}
$$

Let us note that we have the following relations after the final rotation scheme:

$$
\begin{aligned}
& \Sigma^{n+1}=\mathbf{R}_{n}^{n+1} \Sigma^{\mathbf{c}^{n+1}} \mathbf{R}_{n}^{n+1^{\mathrm{T}}} \\
& \mathbf{N}^{n+1}=\mathbf{R}_{n}^{n+1} \mathbf{N}^{c^{n+1}} \mathbf{R}_{n}^{n+1^{\mathrm{T}}} \\
& \boldsymbol{\alpha}^{n+1}=\mathbf{R}_{n}^{n+1} \boldsymbol{\alpha}^{\boldsymbol{c}^{n+1}} \mathbf{R}_{n}^{n+1^{\mathrm{T}}}
\end{aligned}
$$

Now we will establish the expression for the internal forces in terms of the Cauchy stress tensor.

\subsection{Internal forces formulation}

Let $V \subset \mathbb{R}^{3}$ be the volume of the body and $S$ be the surface bounding $V$. Each nodes of $V$ is referenced by its initial positions $\vec{x}_{0} \in \mathbb{R}^{3}$. The initial velocities are denoted $\dot{\vec{x}}_{0}\left(\vec{x}_{0}\right)$. Surface $S$ is decomposed into a part $S_{\vec{x}}$ where the displacements are imposed and a part $S_{\vec{t}}$ where the loads are imposed (could be imposed to be null). We always have $S_{\vec{x}} \cup S_{\vec{t}}=S$ and $S_{\vec{x}} \cap S_{\vec{t}}=0$.

Let $t$ be the time and $\mathscr{T}=\left[0, t_{f}\right]$ be the time interval of integration. Let $\overrightarrow{\vec{x}}(t)$ be the imposed positions on the part $S_{\vec{x}}$. Let $\delta \vec{x}\left(\vec{x}_{0}, t\right)$ be an admissible virtual trajectory with $\delta \vec{x}\left(\vec{x}_{0}, t\right)=0$ for $\vec{x}_{0} \in S_{\vec{x}}$ and $\delta \vec{x}\left(\vec{x}_{0}, t=0\right)=0$ and $\delta \vec{x}\left(\vec{x}_{0}, t=t_{f}\right)=0$ for each $\vec{x}_{0} \in V_{0}$. Let $\vec{b}$ be the volumetric forces and $\vec{t}$ be the surface tractions imposed on $S_{\vec{t}}$.

Then the following quasi-variational principle (principle of virtual power of forces) must hold (Antman, 1995):

$$
\int_{0}^{t_{f}}\left\{\int_{V}\left[\rho \dot{\vec{x}} \cdot \delta \dot{\vec{x}}-\Sigma^{\mathrm{T}}: \frac{\partial \delta \vec{x}}{\partial \vec{x}}+\rho \vec{b} \cdot \delta \vec{x}\right] \mathrm{d} V+\int_{S_{\vec{t}}}[\vec{t} \cdot \delta \vec{x}] \mathrm{d} S\right\} \mathrm{d} t=0 \quad \forall \delta \vec{x} \text { admissible }
$$

where operation $\vec{a} \cdot \vec{b}$ is defined by $\vec{a}_{i} \vec{b}_{i}$. Integrating by part, one gets

$$
\int_{0}^{t_{f}}\left\{\int_{V}\left[\rho \ddot{\vec{x}} \cdot \delta \vec{x}+\Sigma^{\mathrm{T}}: \frac{\partial \delta \vec{x}}{\partial \vec{x}}-\rho \vec{b} \cdot \delta \vec{x}\right] \mathrm{d} V-\int_{S_{\vec{t}}}[\vec{t} \cdot \delta \vec{x}] \mathrm{d} S\right\} \mathrm{d} t=0 \quad \forall \delta \vec{x} \text { admissible }
$$

Therefore, it leads to

$$
\delta K^{n}+\delta W_{\mathrm{int}}^{n}=\delta W_{\mathrm{ext}}^{n} \quad \forall t \in\left[0, t_{f}\right]
$$

with $\delta W_{\text {int }}, \delta W_{\text {ext }}$ and $\delta K$ respectively the virtual work of internal forces, the virtual work of external forces and the virtual work of inertia forces, defined by

$$
\begin{aligned}
& \delta K=\int_{V}\{\rho \ddot{\vec{x}} \cdot \delta \vec{x}\} \mathrm{d} V \\
& \delta W_{\text {ext }}=\int_{V}\{\rho \vec{b} \cdot \delta \vec{x}\} \mathrm{d} V+\int_{S_{\vec{t}}}\{\vec{t} \cdot \delta \vec{x}\} \mathrm{d} S \\
& \delta W_{\text {int }}=\int_{V}\left\{\Sigma^{\mathrm{T}}: \frac{\partial \delta \vec{x}}{\partial \vec{x}}\right\} \mathrm{d} V
\end{aligned}
$$

To integrate Eq. (23) through time, the time interval $\mathscr{T}$ is decomposed into partitions $\left[t_{n}, t_{n+1}\right]$ (such as $\left.\mathscr{T}=\bigcup_{n=0}^{N}\left[t_{n}, t_{n+1}\right]\right)$ with $\Delta t=t_{n+1}-t_{n}$ the time step size. Therefore, superscripts $n$ and $n+1$ are respectively used to denote a value at time $t_{n}$ or at time $t_{n+1}$. Using (1), (6), the mass conservation law (i.e. $\left.\rho^{n} \mathrm{~d} V_{n}=\rho^{0} \mathrm{~d} V_{0}\right)$ and the spatial discretization of the virtual displacement (i.e. $\delta \vec{x}=\varphi^{\xi} \delta \vec{x}^{\xi}$ ), the virtual work of the inertia forces at time $t_{n}$ can be rewritten as 


$$
\delta K^{n}=\int_{V_{0}}\left\{\rho^{0} \varphi^{\xi} \varphi^{\mu}\right\} \mathrm{d} V_{0}\left[\ddot{\vec{x}}^{n}\right]^{\mu} \cdot \delta \vec{x}^{\xi}=M^{\xi \mu}\left[\ddot{\vec{x}}^{n}\right]^{\mu} \cdot \delta \vec{x}^{\xi}
$$

where $M^{\xi \mu}$ is the mass matrix component relative to nodes $\xi$ and $\mu$. The virtual work of external forces is expressed as

$$
\delta W_{\mathrm{ext}}^{n}=\int_{V_{0}}\left\{\rho_{0} \vec{b}^{n} \varphi^{\xi}\right\} \mathrm{d} V_{0} \cdot \delta \vec{x}^{\xi}+\int_{S_{\vec{t}}^{n}}\left\{\vec{t}^{n} \varphi_{\mathrm{S}}^{\xi}\right\} \mathrm{d} S \cdot \delta \vec{x}^{\xi}=\left[\vec{F}_{\mathrm{ext}}^{n}\right]^{\xi} \cdot \delta \vec{x}^{\xi}
$$

with $\varphi_{\mathrm{S}}$ the shape functions of the surface. Finally, the internal forces variation can be rewritten as

$$
\delta W_{\text {int }}^{n}=\int_{V_{0}}\left\{\Sigma^{n^{\mathrm{T}}}\left[\frac{\partial \varphi^{\xi}}{\partial \vec{x}^{n}}\right]^{\mathrm{T}} J_{0}^{n}\right\} \mathrm{d} V_{0} \cdot \delta \vec{x}^{\xi}=\int_{V_{0}}\left\{\Sigma^{n^{\mathrm{T}}} \mathbf{f}_{0}^{n^{\mathrm{T}}} \vec{D}^{\xi} J_{0}^{n}\right\} \mathrm{d} V_{0} \cdot \delta \vec{x}^{\xi}
$$

where $\vec{D}$ is the derivative of the shape function (in the reference configuration, i.e. $\vec{D}^{\xi}=\frac{\partial \varphi^{\xi}}{\partial \vec{x}^{0}}$ ).

Using relations (25)-(27), the balance equation at node $\xi$ for the configuration $n$ leads to

$$
M^{\xi \mu}\left[\ddot{\vec{x}}^{n}\right]^{\mu}=\left[\vec{F}_{\text {ext }}^{n}-\vec{F}_{\text {int }}^{n}\right]^{\xi}
$$

with the expression of internal forces given by

$$
\left[\vec{F}_{\text {int }}^{n}\right]^{\xi}=\int_{V_{0}}\left\{\Sigma^{n^{\mathrm{T}}} \mathbf{f}_{0}^{n^{\mathrm{T}}} \vec{D}^{\xi} J_{0}^{n}\right\} \mathrm{d} V_{0}
$$

\section{The energy momentum conserving scheme}

First the mid-point scheme presented by Simo and Tarnow (1992) is briefly recalled. We explain how to solve it with a predictor-corrector algorithm using Newton-Raphson iteration associated with a line search algorithm. Next an internal force formulation is proposed for an hypoelastic model verifying the conservation laws. The implementation of this formulation for a pressure under-integrated element is also explained.

\subsection{The mid-point scheme}

For an integration from time $t_{n}$ to time $t_{n}+\Delta t=t_{n+1}$, the relations between positions, velocities and accelerations are given by

$$
\begin{aligned}
& \vec{x}^{n+\frac{1}{2}}=\frac{\vec{x}^{n+1}+\vec{x}^{n}}{2} \\
& \dot{\vec{x}}^{n+\frac{1}{2}}=\frac{\vec{x}^{n+1}-\vec{x}^{n}}{\Delta t}=\frac{\dot{\vec{x}}^{n+1}+\dot{\vec{x}}^{n}}{2} \\
& \ddot{\vec{x}}^{n+\frac{1}{2}}=\frac{\dot{\vec{x}}^{n+1}-\dot{\vec{x}}^{n}}{\Delta t}=\frac{\ddot{\vec{x}}^{n+1}+\ddot{\vec{x}}^{n}}{2}
\end{aligned}
$$

These relations give $\dot{\vec{x}}^{n+\frac{1}{2}}, \dot{\vec{x}}^{n+1}, \ddot{\vec{x}}^{n+\frac{1}{2}}$ and $\ddot{\vec{x}}^{n+1}$ from values at time $t_{n}$ and from $\vec{x}^{n+1}$. Positions $\vec{x}^{n+1}$ are obtained from the balance law for node $\xi$ that is applied at the fractional step $n+\frac{1}{2}$,

$$
M^{\xi \mu}\left[\ddot{\vec{x}}^{n+\frac{1}{2}}\right]^{\mu}=\left[\vec{F}_{\mathrm{ext}}^{n+\frac{1}{2}}-\vec{F}_{\mathrm{int}}^{n+\frac{1}{2}}\right]^{\xi}
$$


Let $\vec{F}^{n+\frac{1}{2}}\left(\vec{x}^{n}, \vec{x}^{n+1}\right)$ be the expression of the forces in configuration $n+\frac{1}{2}$. This expression depends both on the position in configuration $n$ (i.e. $\vec{x}^{n}$ ) and $n+1$ (i.e. $\vec{x}^{n+1}$ ). The goal of the following section is to evaluate it for hypoelastic models. The system (30) and (31) can be resolved by a predictor-corrector algorithm. The predicted values (denoted iteration 0 at configuration $n+1)$ are obtained from the relations (30) with $\ddot{\vec{x}}^{n+1}$ supposed to be null (these are generally the most efficient values (Géradin and Rixen, 1994; Géradin and Cardona, 2000) to reach the balanced solution)

$$
\begin{aligned}
& \vec{x}^{n+1,0}=\vec{x}^{n}+\Delta t \dot{\vec{x}}^{n}+\frac{\Delta t^{2}}{4} \ddot{\vec{x}}^{n} \\
& \dot{\vec{x}}^{n+1,0}=\dot{\vec{x}}^{n}+\frac{\Delta t}{2} \ddot{\vec{x}}^{n} \\
& \ddot{\vec{x}}^{n+1,0}=0
\end{aligned}
$$

Residual for Newton-Raphson iteration $i$ at configuration $n+1$ is expressed as

$$
\Delta \vec{F}^{i^{\xi}}=\frac{1}{2} M^{\xi \mu}\left[\ddot{\vec{x}}^{n+1, i}+\ddot{\vec{x}}^{n}\right]^{\mu}+\left[\vec{F}_{\text {int }}^{n+\frac{1}{2}}\left(\vec{x}^{n+1, i}\right)-\vec{F}_{\mathrm{ext}}^{n+\frac{1}{2}}\left(\vec{x}^{n+1, i}\right)\right]^{\xi}
$$

Then, the corrections for iteration $i+1$ at configuration $n+1$ are evaluated as

$$
\begin{aligned}
& {\left[\mathbf{K}^{\xi \mu^{i}}+\frac{2}{\Delta t^{2}} M^{\xi \mu} \mathbf{I}\right] \Delta \vec{x}^{i+1^{\mu}}=-\Delta \vec{F}^{i^{\xi}}} \\
& {\left[\vec{x}^{n+1, i+1}\right]^{\mu}=\left[\vec{x}^{n+1, i}+\alpha_{1 \mathrm{~s}} \Delta \vec{x}^{i+1}\right]^{\mu}} \\
& {\left[\dot{\vec{x}}^{n+1, i+1}\right]^{\mu}=\left[\dot{\vec{x}}^{n+1, i}+\frac{2}{\Delta t} \alpha_{\mathrm{ls}} \Delta \vec{x}^{i+1}\right]^{\mu}} \\
& {\left[\ddot{\vec{x}}^{n+1, i+1}\right]^{\mu}=\left[\ddot{\vec{x}}^{n+1, i}+\frac{4}{\Delta t^{2}} \alpha_{1 \mathrm{~s}} \Delta \vec{x}^{i+1}\right]^{\mu}}
\end{aligned}
$$

where $\mathbf{K}^{\xi \mu}$ is the tangent stiffness matrix

$$
\mathbf{K}^{\xi \mu}=\frac{\partial\left[\vec{F}_{\text {int }}^{n+\frac{1}{2}}\right]^{\xi}}{\partial\left[\vec{x}^{n+1}\right]^{\mu}}-\frac{\partial\left[\vec{F}_{\text {ext }}^{n+\frac{1}{2}}\right]^{\xi}}{\partial\left[\vec{x}^{n+1}\right]^{\mu}}
$$

and $\alpha_{1 \mathrm{~s}}$ is the line search parameter reached by an iterative system (Matthies and Strang, 1979). The convergence of the line search system is obtained when the following relation is verified $\left(\mathrm{Tol}_{\mathrm{ls}}\right.$ is the tolerance specified for each numerical example):

$$
\left\|\Delta \vec{F}^{\xi}\left(\vec{x}^{n+1, i}+\alpha_{\mathrm{ls}} \Delta \vec{x}^{i+1}\right) \cdot \Delta \vec{x}^{i+1}{ }^{\xi}\right\|<\mathrm{Tol}_{\mathrm{ls}}
$$

Eqs. (34) are solved iteratively until convergence of the iterations occurs, i.e. until

$$
\frac{\left.\Delta \vec{F}^{i+1^{\xi}} \cdot \Delta \vec{F}^{i+1}\right]^{\xi}}{\left[\vec{F}_{\text {int }}^{n+\frac{1}{2}}\left(x^{n+1}\right)\right]^{\xi} \cdot\left[\vec{F}_{\text {int }}^{n+\frac{1}{2}}\left(\vec{x}^{n+1}\right)\right]^{\xi}+\left[\vec{F}_{\text {ext }}^{n+\frac{1}{2}}\left(\vec{x}^{n+1}\right)\right]^{\xi} \cdot\left[\vec{F}_{\text {ext }}^{n+\frac{1}{2}}\left(\vec{x}^{n+1}\right)\right]^{\xi}}<\text { Tol }
$$

where Tol is a user defined tolerance specified for each numerical example. In Eq. (31) the explicit form of $\vec{F}_{\text {int }}^{n+\frac{1}{2}}$ has not been given. In the subsequent section an expression for $\vec{F}_{\text {int }}^{n+\frac{1}{2}}$ will be tailored so that it verifies the conservation conditions. 


\subsection{The internal forces expression for hypoelastic materials}

If the expression of the internal forces is evaluated at $\vec{x}^{n+\frac{1}{2}}$, the volume will then be evaluated for an intermediate configuration that will introduce a parasitic volume change. Moreover, the conservation relations are generally not verified. Therefore, the following expression for $\left[\vec{F}_{\text {int }}^{n+\frac{1}{2}}\right]$ is proposed

$$
\begin{aligned}
& {\left[\vec{F}_{\text {int }}^{n+\frac{1}{2}}\right]^{\xi}=\frac{1}{2}\left[\vec{F}_{\text {int }}^{*}+\vec{F}_{\text {int }}^{* *}\right]^{\xi}} \\
& {\left[\vec{F}_{\text {int }}^{*}\right]^{\xi}=\frac{1}{2} \int_{V_{0}}\left\{\left[\mathbf{I}+\mathbf{F}_{n}^{n+1}\right]\left[\Sigma^{n^{\mathrm{T}}}+\mathbf{C}^{*}\right] \mathbf{f}_{0}^{n^{\mathrm{T}}} \vec{D}^{\xi} J_{0}^{n}\right\} \mathrm{d} V_{0}} \\
& {\left[\vec{F}_{\text {int }}^{* *}\right]^{\xi}=\frac{1}{2} \int_{V_{0}}\left\{\left[\mathbf{I}+\mathbf{f}_{n}^{n+1}\right]\left[\Sigma^{n+1^{\mathrm{T}}}+\mathbf{C}^{* *}\right] \mathbf{f}_{0}^{n+1^{\mathrm{T}}} \vec{D}^{\xi} J_{0}^{n+1}\right\} \mathrm{d} V_{0}}
\end{aligned}
$$

where $\mathbf{C}^{*}$ and $\mathbf{C}^{* *}$ are two symmetric tensors that are equal to zero if no plastic deformation occurs, and that will be determined later if plastic deformation occurs. These tensors were neglected in our previous work (Noels et al., 2004). The stress tensors are evaluated by the final rotation scheme combined with the radial return mapping (see Section 2.2). The stress tensor in configuration $n+1$ is evaluated from the stress tensor in configuration $n$. Therefore, the scheme remains incrementally objective. Moreover, in relation (38), the stress tensors are always integrated over their respective volume (through $J$ ). Therefore, no parasitic volume variations are induced. Now, we will demonstrate that relation (38) verifies the conservation laws.

Eq. (31) has to verify the linear and angular momentum conservation, and the energy balance. The first two conditions result from the physical laws assuming that the internal forces cannot change the rigid motion of a body. The last condition assumes that the total mechanic (kinematic and potential) energy of a system without external loading is preserved for a reversible transformation and is decreasing for an irreversible transformation.

\subsubsection{The linear momentum conservation}

Let $\vec{L}$ be the vector (first order tensor) discrete linear momentum

$$
\vec{L} \equiv \sum_{\xi} M^{\xi \mu} \dot{\vec{x}}^{\mu}
$$

where we have kept the convention of summing over repeated indices. The conservation of $\vec{L}$ over a time step is discretized in

$$
\vec{L}^{n+1}-\vec{L}^{n}=\Delta t \sum_{\xi}\left[\vec{F}_{\text {ext }}^{n+\frac{1}{2}}\right]^{\xi}
$$

By performing a sum over $\xi$ in Eq. (31), and using (30), it leads to

$$
\begin{aligned}
& \frac{1}{\Delta t} \sum_{\xi} M^{\xi \mu}\left[\dot{\vec{x}}^{n+1}-\dot{\vec{x}}^{n}\right]^{\mu}=\sum_{\xi}\left[\vec{F}_{\mathrm{ext}}^{n+\frac{1}{2}}-\vec{F}_{\mathrm{int}}^{n+\frac{1}{2}}\right]^{\xi} \\
& \frac{1}{\Delta t}\left[\vec{L}^{n+1}-\vec{L}^{n}\right]=\sum_{\xi}\left[\vec{F}_{\mathrm{ext}}^{n+\frac{1}{2}}-\vec{F}_{\mathrm{int}}^{n+\frac{1}{2}}\right]^{\xi}
\end{aligned}
$$

But, using the following properties of the shape functions $(\forall i \in[1,3])$

$$
\sum_{\xi} \vec{D}_{i}^{\xi}=\sum_{\xi} \frac{\partial \varphi^{\xi}}{\partial \vec{x}_{i}^{0}}=0
$$


and the definition of $\vec{F}_{\text {int }}^{n+\frac{1}{2}}$ (38), the relation (41) is reduced to (40), which proves the conservation of the linear momentum.

\subsubsection{The angular momentum conservation}

Let $\vec{J}$ be the first order tensor (vector) discrete angular momentum defined by the following vector product:

$$
\vec{J} \equiv M^{\xi \mu}\left[\vec{x}^{\xi} \wedge \dot{\vec{x}}^{\mu}\right]
$$

The conservation of $\vec{J}$ over a time step is discretized in

$$
\vec{J}^{n+1}-\vec{J}^{n}=\Delta t\left[\vec{x}^{n+\frac{1}{2}}\right]^{\xi} \wedge\left[\vec{F}_{\text {ext }}^{n+\frac{1}{2}}\right]^{\xi}
$$

The vector product of $\vec{x}^{n+\frac{1}{2}}$ by relation (31) gives

$$
M^{\xi \mu}\left[\vec{x}^{n+\frac{1}{2}}\right]^{\xi} \wedge\left[\ddot{\vec{x}}^{n+\frac{1}{2}}\right]^{\mu}=\left[\vec{x}^{n+\frac{1}{2}}\right]^{\xi} \wedge\left[\vec{F}_{\text {ext }}^{n+\frac{1}{2}}-\vec{F}_{\text {int }}^{n+\frac{1}{2}}\right]^{\xi}
$$

Using relations (30), this last expression leads to

$$
\begin{aligned}
& \frac{1}{\Delta t} M^{\xi \mu}\left\{\left[\vec{x}^{n+1}\right]^{\xi} \wedge\left[\dot{\vec{x}}^{n+1}\right]^{\mu}-\left[\vec{x}^{n}\right]^{\xi} \wedge\left[\dot{\vec{x}}^{n}\right]^{\mu}\right\}=\left[\vec{x}^{n+\frac{1}{2}}\right]^{\xi} \wedge\left[\vec{F}_{\mathrm{ext}}^{n+\frac{1}{2}}-\vec{F}_{\mathrm{int}}^{n+\frac{1}{2}}\right]^{\mu} \\
& \frac{1}{\Delta t}\left[\vec{J}^{n+1}-\vec{J}^{n}\right]=\left[\vec{x}^{n+\frac{1}{2}}\right]^{\xi} \wedge\left[\vec{F}_{\mathrm{ext}}^{n+\frac{1}{2}}\right]^{\xi}-\left[\vec{x}^{n+\frac{1}{2}}\right]^{\xi} \wedge\left[\vec{F}_{\mathrm{int}}^{n+\frac{1}{2}}\right]^{\xi}
\end{aligned}
$$

Let us consider $\left(\vec{F}_{\text {int }}^{*}\right)_{i}^{\xi}$ from relation (38). Let $\epsilon$ be the third order permutation tensor such that, for each vector $\vec{a}$ and $\vec{b}$, it comes $(\vec{a} \wedge \vec{b})=\epsilon:[\vec{a} \otimes \vec{b}]$, with operation $[\vec{a} \otimes \vec{b}]_{i j}=\vec{a}_{i} \vec{b}_{j}$. Therefore, it leads to

$$
\begin{aligned}
2\left[\vec{x}^{n+\frac{1}{2}}\right]^{\xi} \wedge\left[\vec{F}_{\text {int }}^{*}\right]^{\xi} & =\epsilon:\left\{\left[\vec{x}^{n+1}+\vec{x}\right]^{\xi} \otimes\left[\vec{F}_{\text {int }}^{*}\right]^{\xi}\right\} \\
& =\frac{1}{2} \epsilon:\left\{\left[\vec{x}^{n+1}+\vec{x}^{n}\right]^{\xi} \otimes \int_{V_{0}}\left\{\left[\mathbf{I}+\mathbf{F}_{n}^{n+1}\right]\left[\Sigma^{n^{\mathrm{T}}}+\mathbf{C}^{*}\right] \mathbf{f}_{0}^{n+1^{\mathrm{T}}} \vec{D}^{\xi} J_{0}^{n}\right\} \mathrm{d} V_{0}\right\}
\end{aligned}
$$

Using (2) and (4) yields

$$
\begin{aligned}
& {\left[\vec{x}^{n}\right]^{\xi} \otimes\left[\mathbf{f}_{0}^{\mathrm{n}^{\mathrm{T}}} \vec{D}^{\xi}\right]=\mathbf{I}} \\
& {\left[\vec{x}^{n+1}\right]^{\xi} \otimes\left[\mathbf{f}_{0}^{n^{\mathrm{T}}} \vec{D}^{\xi}\right]=\mathbf{F}_{n}^{n+1^{\mathrm{T}}}}
\end{aligned}
$$

Thanks to relations (48) and the fact that $\Sigma$ is symmetric, relation (47) becomes

$$
4\left[\vec{x}^{n+\frac{1}{2}}\right]^{\xi} \wedge\left[\vec{F}_{\text {int }}^{*}\right]^{\xi}=\epsilon: \int_{V_{0}}\left\{\left[\mathbf{I}+\mathbf{F}_{n}^{n+1}\right]\left[\boldsymbol{\Sigma}^{n}+\mathbf{C}^{*}\right]\left[\mathbf{I}+\mathbf{F}_{n}^{n+1}\right]^{\mathrm{T}} J_{0}^{n}\right\} \mathrm{d} V_{0}=\int_{V_{0}}\left\{\epsilon: \boldsymbol{\Theta} J_{0}^{n}\right\} \mathrm{d} V_{0}=0
$$

where $\boldsymbol{\Theta}=\left[\mathbf{I}+\mathbf{F}_{n}^{n+1}\right]\left[\boldsymbol{\Sigma}^{n}+\mathbf{C}^{*}\right]\left[\mathbf{I}+\mathbf{F}_{n}^{n+1}\right]^{\mathrm{T}}$ is a symmetric tensor (assuming that both $\boldsymbol{\Sigma}$ and $\mathbf{C}^{*}$ are symmetric tensors), and $\epsilon$ is an anti-symmetric third order tensor. Therefore $\epsilon: \boldsymbol{\Theta}$ is equal to zero. The same process with $\vec{F}_{\text {int }}^{* *}$ also leads to

$$
\left[\vec{x}^{n+\frac{1}{2}}\right]^{\xi} \wedge\left[\vec{F}_{\text {int }}^{* *}\right]^{\xi}=0
$$

Finally, using relations (48) and (49), Eq. (46) is reduced to (44) and the conservation of the angular momentum is verified. 


\subsubsection{The energy balance}

Let $E, W_{\text {int }}, W_{\text {ext }}$ and $K$ respectively be the total energy, the internal energy, the external energy and the kinematic energy. It comes

$$
E=K+W_{\text {int }}-W_{\text {ext }}
$$

The energy balance over one time step is discretized in

$$
E^{n+1}-E^{n}=-\Delta_{\text {int }}
$$

with $\Delta_{\text {int }} \geqslant 0$ the dissipation during the time step from configuration $n$ to $n+1$. The scalar product of $\dot{\vec{x}}^{n+\frac{1}{2}}$ and of relation (31) leads to (using relation (30))

$$
\begin{aligned}
M^{\xi \mu}\left[\ddot{\vec{x}}^{n+\frac{1}{2}}\right]^{\mu} \cdot\left[\dot{\vec{x}}^{n+\frac{1}{2}}\right]^{\xi}= & {\left[\vec{F}_{\text {ext }}^{n+\frac{1}{2}}-\vec{F}_{\text {int }}^{n+\frac{1}{2}}\right]^{\xi} \cdot\left[\overrightarrow{\vec{x}}^{n+\frac{1}{2}}\right]^{\xi} } \\
& \times \frac{M^{\xi \mu}}{2 \Delta t}\left\{\left[\dot{\vec{x}}^{n+1}\right]^{\xi} \cdot\left[\dot{\vec{x}}^{n+1}\right]^{\mu}-\left[\dot{\vec{x}}^{n}\right]^{\xi} \cdot\left[\dot{\vec{x}}^{n}\right]^{\mu}\right\}=\frac{1}{\Delta t}\left[\vec{F}_{\mathrm{ext}}^{n+\frac{1}{2}}-\vec{F}_{\mathrm{int}}^{n+\frac{1}{2}}\right]^{\xi} \cdot\left[\vec{x}^{n+1}-\vec{x}^{n}\right]^{\xi}
\end{aligned}
$$

or

$$
K^{n+1}-K^{n}+\left[\vec{F}_{\mathrm{int}}^{n+\frac{1}{2}}\right]^{\xi} \cdot\left[\vec{x}^{n+1}-\vec{x}^{n}\right]^{\xi}=W_{\mathrm{ext}}^{n+1}-W_{\mathrm{ext}}^{n}
$$

First $\vec{F}_{\text {int }}^{*}$ (38) is included in (59). Using (2) and (4), it comes

$$
\begin{aligned}
{\left[\vec{x}^{n+1}-\vec{x}^{n}\right]^{\xi} \cdot\left[\vec{F}_{\text {int }}^{*}\right]^{\xi} } & =\frac{1}{2}\left[\vec{x}^{n+1}-\vec{x}^{n}\right]^{\xi} \cdot \int_{V_{0}}\left\{\left[\mathbf{I}+\mathbf{F}_{n}^{n+1}\right]\left[\boldsymbol{\Sigma}^{n}+\mathbf{C}^{*}\right] \mathbf{f}_{0}^{n^{\mathrm{T}}} \vec{D}^{\xi} J_{0}^{n}\right\} \mathrm{d} V_{0} \\
& =\frac{1}{2} \int_{V_{0}}\left\{\left[\mathbf{F}_{n}^{n+1^{\mathrm{T}}} \mathbf{F}_{n}^{n+1}+\mathbf{F}_{n}^{n+1}-\mathbf{F}_{n}^{n+1^{\mathrm{T}}}-\mathbf{I}\right]:\left[\boldsymbol{\Sigma}^{n}+\mathbf{C}^{*}\right] J_{0}^{n}\right\} \mathrm{d} V_{0}
\end{aligned}
$$

Since $\boldsymbol{\Sigma}+\mathbf{C}^{*}$ is symmetric, one has

$$
\mathbf{F}_{n}^{n+1^{\mathrm{T}}}:\left[\boldsymbol{\Sigma}^{n}+\mathbf{C}^{*}\right]-\mathbf{F}_{n}^{n+1}:\left[\boldsymbol{\Sigma}^{n}+\mathbf{C}^{*}\right]=0
$$

Using (7) and (56), the relation (55) becomes

$$
\left[\vec{x}^{n+1}-\vec{x}^{n}\right]^{\xi} \cdot\left[\vec{F}_{\text {int }}^{*}\right]^{\xi}=\int_{V_{0}}\left\{\mathbf{G L}_{n}^{n+1}:\left[\boldsymbol{\Sigma}^{n}+\mathbf{C}^{*}\right] J_{0}^{n}\right\} \mathrm{d} V_{0}
$$

For $\vec{F}_{\text {int }}^{* *}$ the same process leads to

$$
\left[\vec{x}^{n+1}-\vec{x}^{n}\right]^{\xi} \cdot\left[\vec{F}_{\text {int }}^{* *}\right]^{\xi}=\int_{V_{0}}\left\{\mathbf{A}_{n}^{n+1}:\left[\boldsymbol{\Sigma}^{n+1}+\mathbf{C}^{* *}\right] J_{0}^{n+1}\right\} \mathrm{d} V_{0}
$$

and finally, using relations (54), (57) and (58), the relation of the energy conservation (52) is reduced to

$$
W_{\text {int }}^{n+1}-W_{\text {int }}^{n}+\Delta_{\text {int }}=\frac{1}{2} \int_{V_{0}}\left\{\mathbf{G L}_{n}^{n+1}:\left[\boldsymbol{\Sigma}^{n}+\mathbf{C}^{*}\right] J_{0}^{n}+\mathbf{A}_{n}^{n+1}:\left[\boldsymbol{\Sigma}^{n+1}+\mathbf{C}^{* *}\right] J_{0}^{n+1}\right\} \mathrm{d} V_{0}
$$

This last equation is the one which should be verified if we require the time integration algorithm to be energy conserving. From this point, for a hyperelastic material, a potential $\phi$ could be written to evaluate the internal energy as demonstrated by Meng and Laursen (2001). However, for a hypoelastic material, no potential can be defined unless the transformations are supposed to be infinitesimal. In this special case, relation (59) is directly verified as demonstrated by Noels et al. (2004). In the large transformation case, we will proceed as follow. Let us imagine a virtual loading-unloading cycle, that takes place in two steps, from configuration 1 to 2 and then from 2 to 3 (Fig. 1), such that initial Cauchy stress tensor $\Sigma^{1}$ corresponds to final Cauchy stress tensor $\Sigma^{3}$ up to any arbitrary rotation $\mathbf{Q}\left(\mathbf{Q}^{\mathrm{T}} \mathbf{Q}=\mathbf{I}\right.$ and $\left.\operatorname{det} \mathbf{Q}=1\right)$, 


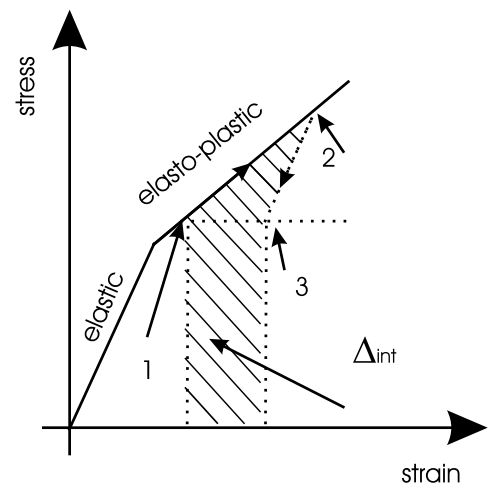

Fig. 1. Definition of the loading-unloading cycle (1D analogy).

$$
\Sigma^{3}=\mathbf{Q} \Sigma^{1} \mathbf{Q}^{\mathrm{T}}
$$

During the loading phase from configuration 1 to 2, we assume that plastic deformations occur, while the transition from configuration 2 to configuration 3 corresponds to elastic unloading. Note that configuration 3 might be kinematically inadmissible for a whole body, but this is not of concern here since we are reasoning at the elementary volume level. The expression of internal forces (38) is consistent with the Druckers Postulate (e.g. Lubliner, 1990) if the reversible work of the loading phase is recovered during the second step (i.e. $W_{\text {int }}^{3}-W_{\text {int }}^{1}=0$ ). Therefore, if we assume that tensors $\mathbf{C}^{*}$ and $\mathbf{C}^{* *}$ are equal to zero if no plastic deformation occur (i.e. during the second step), the energy balance (59) between the configurations 1 and 3 can be expressed as

$$
\Delta_{\text {int }}=\Delta_{\text {int } 1}^{3}=\frac{1}{2} \int_{V_{0}}\left\{\mathbf{G L}_{1}^{2}:\left[\boldsymbol{\Sigma}^{1}+\mathbf{C}^{*}\right] J_{0}^{1}+\mathbf{A}_{1}^{2}:\left[\boldsymbol{\Sigma}^{2}+\mathbf{C}^{* *}\right] J_{0}^{2}\right\} \mathrm{d} V_{0}+\frac{1}{2} \int_{V_{0}}\left\{\mathbf{G L}_{2}^{3}: \Sigma^{2} J_{0}^{2}+\mathbf{A}_{2}^{3}: \Sigma^{3} J_{0}^{3}\right\} \mathrm{d} V_{0}
$$

Let $\mathbf{E}_{1}^{\text {el2 }}$ be the elastic natural strain tensor defined such that

$$
\mathscr{H}: \mathbf{E}_{1}^{\mathrm{el} 2} \equiv \mathscr{H}: \mathbf{E}_{1}^{2}-\mathbf{s}_{1}^{c 2}
$$

Therefore we define $\mathbf{U}_{1}^{\mathrm{el} 2}$ the symmetric tensor such that

$$
\mathbf{E}_{1}^{\mathbf{e} 2} \equiv \frac{1}{2} \ln \left[\mathbf{U}_{1}^{\mathbf{e l} 2} \mathbf{U}_{1}^{\mathbf{e l} 2}\right]
$$

The existence of $\mathbf{U}_{1}^{\mathrm{el} 2}$ result from the symmetry of tensor $\mathbf{E}_{1}^{\mathbf{e l} 2}$. Elastic and plastic Green-Lagrange strain tensor (respectively $\mathbf{G L}{ }_{1}^{\mathbf{e l} 2}$ and $\mathbf{G} \mathbf{L}_{1}^{\mathbf{p l} 2}$ ), as elastic and plastic Almansi strain tensor (respectively $\mathbf{A}_{1}^{\mathbf{e l} 2}$ and $\mathbf{A}_{1}^{\mathbf{p l} 2}$ ) are defined from $\mathbf{U}_{1}^{\mathbf{e} 2}$

$$
\begin{aligned}
& \mathbf{G L}_{1}^{\mathbf{e} 2} \equiv \frac{1}{2}\left[\mathbf{U}_{1}^{\mathbf{e l} 2} \mathbf{U}_{1}^{\mathbf{e} 2}-\mathbf{I}\right] \\
& \mathbf{G L}_{1}^{\mathbf{p} \mathbf{2}} \equiv \mathbf{G L}_{1}^{2}-\mathbf{G L}_{1}^{\mathbf{e} 2} \\
& \mathbf{A}_{1}^{\mathbf{e l} 2} \equiv \frac{1}{2} \mathbf{R}_{1}^{2}\left[\mathbf{I}-\mathbf{U}_{1}^{\mathbf{e} 2^{-1}} \mathbf{U}_{1}^{\mathbf{e} 2^{-1}}\right] \mathbf{R}_{1}^{2^{\mathrm{T}}} \\
& \mathbf{A}_{1}^{\mathbf{p} \mathbf{2} 2} \equiv \mathbf{A}_{1}^{2}-\mathbf{A}_{1}^{\mathbf{e l} 2}
\end{aligned}
$$

As we have demonstrated in (Noels et al., 2004), relation (60), using relations (64) and the assumption that $\mathscr{H}$ is constant, yields 


$$
\begin{aligned}
& \mathbf{G L}_{2}^{3}=-\mathbf{A}_{1}^{\mathbf{e} 2} \\
& \mathbf{A}_{2}^{3}=-\mathbf{Q G L}_{1}^{\mathbf{e} 2} \mathbf{Q}^{\mathrm{T}} \\
& J_{0}^{3}=J_{0}^{1}
\end{aligned}
$$

Therefore, using relations (65), the relation (61) becomes

$$
\Delta_{\text {int }}=\frac{1}{2} \int_{V^{0}}\left\{\left[\mathbf{G L}_{1}^{\mathbf{p} \mathbf{2}}: \boldsymbol{\Sigma}^{1}+\mathbf{G L}_{1}^{2}: \mathbf{C}^{*}\right] J_{0}^{1}+\left[\mathbf{A}_{1}^{\mathbf{p} \mathbf{2}}: \boldsymbol{\Sigma}^{2}+\mathbf{A}_{1}^{2}: \mathbf{C}^{* *}\right] J_{0}^{2}\right\} \mathrm{d} V_{0}
$$

The relation (66) has to be related with a physical quantity. The positive internal plastic dissipation can be expressed from a volumic dissipation $D_{\text {int }}$ as

$$
\Delta_{\text {int }}^{\text {phy }}=\int_{V_{0}}\left\{D_{\text {int }}\right\} \mathrm{d} V_{0}>0
$$

Therefore, we can deduce tensors $\mathbf{C}^{*}$ and $\mathbf{C}^{* *}$ that lead to an energy conserving scheme

$$
\begin{aligned}
& \mathbf{C}^{*}=\frac{\frac{D_{\text {int }}}{J_{0}^{1}}-\Sigma^{1}: \mathbf{G L}_{1}^{\mathbf{p} / 2}}{\mathbf{G L}_{1}^{2}: \mathbf{G L}_{1}^{2}} \mathbf{G L}_{1}^{2} \\
& \mathbf{C}^{* *}=\frac{\frac{D_{\text {int }}}{J_{0}^{2}}-\Sigma^{2}: \mathbf{A}_{1}^{\mathbf{p l} 2}}{\mathbf{A}_{1}^{2}: \mathbf{A}_{1}^{2}} \mathbf{A}_{1}^{2}
\end{aligned}
$$

These tensors are symmetric and are equal to zero when no plastic deformation occurs as assumed. If there are plastic deformations, the dissipation is then equal to $\Delta_{\text {int }}^{\text {phy }}>0$. Moreover, we can prove that these tensors are of second order in the plastic deformation increment. Relation (62) can be transformed, using relation (9), relation (18) and a linearization of the logarithm mapping, as

$$
\begin{aligned}
\mathscr{H} & : \mathbf{E}_{n}^{\mathbf{e} \mathbf{l} n+1}=\mathscr{H}: \mathbf{E}_{n}^{n+1}-\mathbf{s}_{n}^{c n+1} \\
\mathscr{H} & : \mathbf{G L}_{n}^{\mathbf{e} n+1}=\mathscr{H}: \mathbf{G L}_{n}^{n+1}+\mathcal{O}\left(\mathbf{G L}_{n}^{\mathbf{p l} n+1^{2}}\right)-2 g \gamma \mathbf{N}^{\mathbf{c}}
\end{aligned}
$$

or as

$$
\mathscr{H}: \mathbf{A}_{n}^{\mathbf{e l} n+1}=\mathscr{H}: \mathbf{A}_{n}^{n+1}+\mathcal{O}\left(\mathbf{A}_{n}^{\mathbf{p l} n+1^{2}}\right)-2 g \gamma \mathbf{N}
$$

Using the fact that for $J 2$ plasticity the traces of $\mathbf{N}^{\mathbf{c}}$ and of $\mathbf{N}$ are equal to zero, the inversion of Hooke's law (relation (15)) yields

$$
\begin{aligned}
& \mathbf{G L}_{n}^{n+1}-\mathbf{G L}_{n}^{\mathbf{e l} n+1}+\mathcal{O}\left(\mathbf{G L}_{n}^{\mathbf{p} \mathbf{l} n+1^{2}}\right)=\gamma \mathbf{N}^{\mathbf{c}} \\
& \mathbf{A}_{n}^{n+1}-\mathbf{A}_{n}^{\mathbf{e} \mathbf{l} n+1}+\mathcal{O}\left(\mathbf{A}_{n}^{\mathbf{p} \mathbf{l} n+1^{2}}\right)=\gamma \mathbf{N}
\end{aligned}
$$

Therefore, the order of the relations (68) is

$$
\begin{aligned}
& \mathbf{C}^{*}: \mathbf{G L}_{1}^{2} J_{0}^{1}=D_{\text {int }}-\gamma \mathbf{N}^{\mathbf{c}}: \Sigma^{1} J_{0}^{n}+\mathcal{O}\left(\Sigma^{1}: \mathbf{G L}_{1}^{\mathbf{p l} 2^{2}}\right) \\
& \mathbf{C}^{* *}: \mathbf{A}_{1}^{2} J_{0}^{2}=D_{\text {int }}-\gamma \mathbf{N}: \Sigma^{2} J_{0}^{2}+\mathcal{O}\left(\Sigma^{2}: \mathbf{A}_{1}^{\mathbf{p l 1} 2^{2}}\right)
\end{aligned}
$$

But the specific time variation of energy due to the plasticity can be expressed as $\left(\Sigma_{\mathrm{v}}+\bar{\alpha}\right) \dot{\varepsilon}^{\mathrm{p}} / \rho$ (Adam, 2003). In this expression the equivalent heredity $(\bar{\alpha})$ is added to the equivalent von Mises stress to obtain the effective equivalent stress. Therefore, the internal dissipation can be discretized on a time step (assuming linear hardening)

$$
D_{\text {int }}=\frac{1}{2} \epsilon_{n}^{\mathrm{p} n+1}\left\{\left[\sum_{\mathrm{v}}^{n+1}+\bar{\alpha}^{n+1}\right] J_{0}^{n+1}+\left[\sum_{\mathrm{v}}^{n}+\bar{\alpha}^{n}\right] J_{0}^{n}\right\}
$$


With definition of $\gamma(18), \varepsilon(18), \mathbf{N}(17)$ and $\Sigma^{\operatorname{dev}^{n}}$ defining the deviatoric part of $\Sigma^{n}$, the relation (19), at the first order leads to

$$
\begin{aligned}
& \gamma \mathbf{N}^{\mathbf{c}}: \Sigma^{n} J_{0}^{n}=\gamma \mathbf{N}^{\mathbf{c}}: \Sigma^{\operatorname{dev}^{n}} J_{0}^{n} \simeq \varepsilon_{n}^{\mathrm{p} n+1}\left[\Sigma_{\mathrm{v}}^{n}+\bar{\alpha}^{n}\right] J_{0}^{n} \\
& \gamma \mathbf{N}: \Sigma^{n+1} J_{0}^{n+1}=\gamma \mathbf{N}: \Sigma^{\operatorname{dev}^{n+1}} J_{0}^{n+1} \simeq \varepsilon_{n}^{\mathrm{p} n+1}\left[\Sigma_{\mathrm{v}}^{n+1}+\bar{\alpha}^{n+1}\right] J_{0}^{n+1}
\end{aligned}
$$

And finally, the sum of the two terms of expressions (72) leads to second order terms. Therefore, for small increments of transformation, correction tensors $\mathbf{C}^{*}$ and $\mathbf{C}^{* *}$ are of the second order. The consistent tangent stiffness matrix is developed in Appendix A. In this paper we will show the necessity of considering those terms for large time step size increments.

\subsection{Implementation of the internal forces}

The implementation of expression (38) is described for a selective reduced integration (SRI) scheme using one volumic Gauss point and several deviatoric Gauss points. Values referring to the volumic Gauss point are denoted with a superscript $V$ and values relative to a deviatoric Gauss point are denoted with a superscript $D$. The deviatoric part of a tensor is denoted with superscript dev. The pressure of an element is computed and integrated at a single volumic Gauss point to avoid volumic locking. Therefore, the expression of the internal force at this point is (with $p=k \Sigma_{i i}$ )

$$
\left[\vec{F}_{\text {int }}^{n+\frac{1}{2}}\right]^{\xi^{V}}=\frac{1}{4}\left[\mathbf{I}+\mathbf{F}_{n}^{n+1}\right]^{V} p^{n^{V}} \mathbf{I}\left[\mathbf{f}_{0}^{n^{\mathrm{T}}}\right]^{V}\left[\vec{D}^{\xi}\right]^{V} J_{0}^{n^{V}} V_{0}+\frac{1}{4}\left[\mathbf{I}+\mathbf{f}_{n}^{n+1}\right]^{V} p^{n+1^{V}} \mathbf{I}\left[\mathbf{f}_{0}^{n+1^{\mathrm{T}}}\right]^{V}\left[\vec{D}^{\xi}\right]^{V} J_{0}^{n+1^{V}} V_{0}
$$

The deviatoric part of the stress

$$
\Sigma^{\operatorname{dev}^{n+1}}=\mathbf{R}\left[\Sigma^{\operatorname{dev}^{n}}+2 g \mathbf{E}^{\mathrm{dev}}-\mathbf{s}^{\mathbf{c}}\right] \mathbf{R}^{\mathrm{T}}
$$

and the correcting tensors are integrated at the deviatoric Gauss points

$$
\begin{aligned}
{\left[\vec{F}_{\text {int }}^{n+\frac{1}{2}}\right]^{\xi^{D}}=} & \frac{1}{4}\left[\mathbf{I}+\mathbf{F}_{n}^{n+1}\right]^{D}\left[\boldsymbol{\Sigma}^{\operatorname{dev}^{n}}+\mathbf{C}^{*}\right]^{D}\left[\mathbf{f}_{0}^{n^{\mathrm{T}}}\right]^{D}\left[\vec{D}^{\xi}\right]^{D} J_{0}^{n^{D}} V_{0} \\
& +\frac{1}{4}\left[\mathbf{I}+\mathbf{f}_{n}^{n+1}\right]^{D}\left[\boldsymbol{\Sigma}^{\mathbf{d e v}^{n+1}}+\mathbf{C}^{* *}\right]^{D}\left[\mathbf{f}_{0}^{n+1^{\mathrm{T}}}\right]^{D}\left[\vec{D}^{\xi}\right]^{D} J_{0}^{n+1^{D}} V_{0}
\end{aligned}
$$

where the correcting tensors (68) are computed from the work done by the deviatoric stress and the volumic pressure as

$$
\begin{aligned}
& \mathbf{C}^{*}=\frac{\frac{D_{\text {int }}^{D}}{J_{0}^{D}}-\left[\left[\Sigma^{\mathbf{d e v}^{n}}\right]^{D}+p^{n^{V}} \mathbf{I}\right]:\left[\mathbf{G L}_{n}^{\mathbf{p} \mathbf{l}_{n+1}}\right]^{D}}{\left[\mathbf{G L}_{n}^{n+1}\right]^{D}:\left[\mathbf{G L}_{n}^{n+1}\right]^{D}}\left[\mathbf{G L}_{n}^{n+1}\right]^{D} \\
& \mathbf{C}^{* *}=\frac{\frac{D_{\text {int }}^{D}}{J_{0}^{n+1}}-\left[\left[\Sigma^{\mathbf{d e v}^{n+1}}\right]^{D}+p^{n+1}{ }^{V} \mathbf{I}\right]:\left[\mathbf{A}_{n}^{\mathbf{p} \mathbf{l} n+1}\right]^{D}}{\left[A_{n}^{n+1}\right]^{D}:\left[A_{n}^{n+1}\right]^{D}}\left[A_{n}^{n+1}\right]^{D}
\end{aligned}
$$

In these expressions, the plastic tensors are computed from relations (64) with $\mathbf{U}^{\text {el }}$ computed from relations (62) and (63) where all the terms $\left(\mathbf{E}^{\mathrm{el}}, \mathbf{E}\right.$ and $\left.\mathbf{s}^{\mathbf{c}}\right)$ are evaluated at the deviatoric Gauss point $D$. Let us note that for the 2D study of a plane strain deformation problem, the expression (78) is computed taking into account the fact that tensors $\Sigma^{\mathbf{d e v}}, \mathbf{G L}_{n}^{\mathbf{p l n + 1}}$ and $\mathbf{A}_{n}^{\mathbf{p l n} n+1}$ have an out-of-plane component different from zero. 


\section{Numerical examples}

In this section we compare the solution obtained with three methods:

- The results obtained with the proposed conservative scheme (EMCA) taking into account the correcting tensors.

- The results obtained with the proposed conservative scheme (EMCA) without the correcting tensors.

- The Newmark algorithm (Newmark, 1959) (NMK) with the first Newmark parameter $(\beta)$ equal to 0.25 and the second Newmark parameter $(\gamma)$ equal to 0.5 .

For each problem, the time step size is constant to avoid any instabilities resulting from a time step size variation. Each simulation is achieved with different time step sizes to show the influence of the use of the correcting tensors on the stability of the solution.

For the hypoelastic material, the internal energy is not directly accessible. Therefore, the total energy is computed from the work of internal forces. For the conservative algorithm, the total energy at time $t_{n+1}$ is defined as

$$
E^{n+1}=E^{n}+K^{n+1}-K^{n}+\left[\vec{F}_{\text {int }}^{n+\frac{1}{2}}\right]\left[\vec{x}^{n+1}-\vec{x}^{n}\right]-W_{\mathrm{ext}}^{n+1}+W_{\mathrm{ext}}^{n}
$$

with $\vec{F}_{\text {int }}^{n+\frac{1}{2}}$ computed from relation (38). For the Newmark algorithm, it is defined by

$$
E^{n+1}=E^{n}+K^{n+1}-K^{n}+\frac{1}{2}\left[\vec{F}_{\text {int }}^{n}+\vec{F}_{\text {int }}^{n+1}\right]\left[\vec{x}^{n+1}-\vec{x}^{n}\right]-W_{\mathrm{ext}}^{n+1}+W_{\mathrm{ext}}^{n}
$$

with $\vec{F}_{\text {int }}^{n}$ defined from relation (29). This total energy evaluation includes the internal dissipation and must thus remains constant for each problem. The internal dissipation is evaluated from relations (67) and (73). The finite elements used for each example are 8-noded trilinear bricks with eight deviatoric Gauss points and one volumic Gauss point.

\subsection{Example 1: traction/shearing of a $2 D$-element}

First, we will study the influence of the time step size for a problem with no degree of freedom (i.e. where the displacement of all nodes are prescribed). This methodology allows us to evaluate the influence of the correcting terms. It consists of the deformation (traction and shearing) of a plane strain 2D-element. The path of deformation is illustrated in Fig. 2. Properties of the material are reported in Table 1. The simulation occurs in $1 \mathrm{~s}$ and is achieved with five different constant time step sizes $(0.5,0.25,0.1,0.05$ and 0.025 s). Fig. 3(a) illustrates the final value (after $1 \mathrm{~s}$ ) of the work done by the internal forces and Fig. 3(b) illustrates the error (referring to the value obtained with the EMCA algorithm and the correcting tensors for a time step size equal to $0.025 \mathrm{~s}$ ) on the final value. To be pertinent with the order of the correcting tensor, the abscissa axis (logarithmic scale) represents the maximal increment of the equivalent plastic strain during the simulation and not the value of the time step size. Since the error is represented with a logarithmic scale, we can see that the error is of the second order (which is the order of the error of the finite element method integration) and that the most accurate solution is the conservative scheme with the correcting tensors (error lower of $1 \%$ even for an equivalent plastic strain increment of 0.83 ). To have the same error, the time step size (or the maximal equivalent plastic strain increment) must be five times smaller if the correcting tensors are neglected, and must be three times smaller if the Newmark algorithm is used. Once the error of the different algorithms known, we will show the influence of the correcting terms on the stability (convergence rate...) of the integration. 


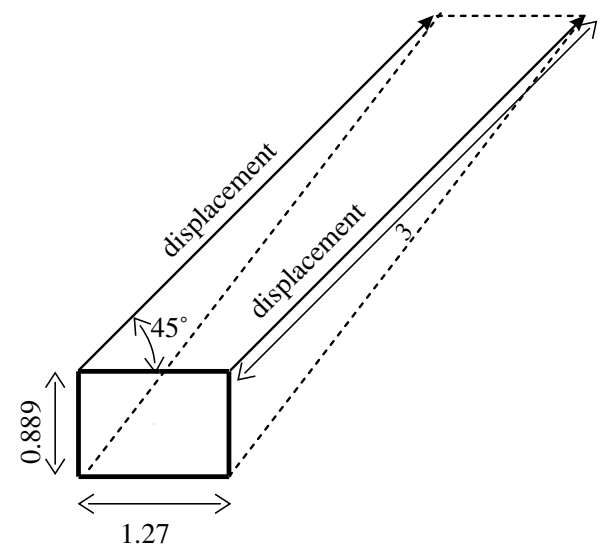

Fig. 2. Deformation of the 2D-element (length in meter): solid lines correspond to initial configuration and dotted lines correspond to final configuration.

Table 1

Material properties of the 2D-element

\begin{tabular}{ll}
\hline Property & Value \\
\hline Density & $\rho=8930 \mathrm{~kg} / \mathrm{m}^{3}$ \\
Young's modulus & $E=206.9 \mathrm{E} 9 \mathrm{~N} / \mathrm{m}^{2}$ \\
Poisson's ratio & $v=0.29$ \\
Yield stress & $\sigma_{0}=450 \mathrm{~N} / \mathrm{mm}^{2}$ \\
Hardening parameter & $h=129.4 \mathrm{~N} / \mathrm{mm}^{2}$ \\
\hline
\end{tabular}

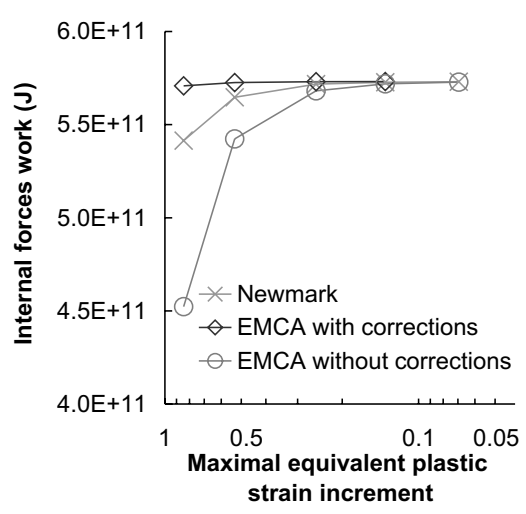

(a)

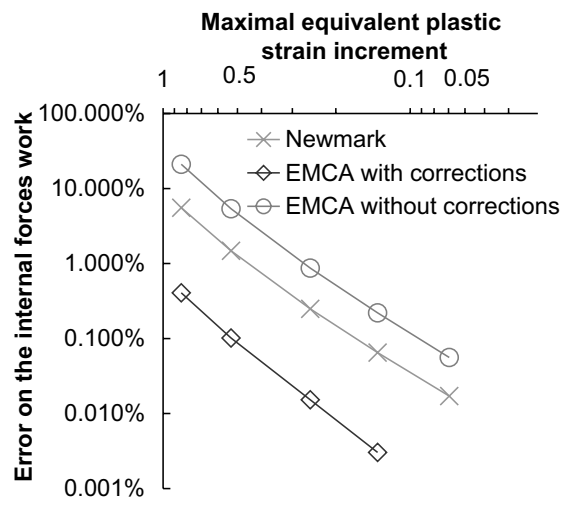

(b)

Fig. 3. Internal forces work: (a) final work, (b) error on the final work computation.

\subsection{Example 2: impact of two cylinders}

This problem, treated here with an hypoelastic material, was first presented by Meng and Laursen (2002) for an hyperelastic material. It consists in one cylinder that impacts, with an initial velocity, another 
Table 2

Geometrical and material properties of the two cylinders

\begin{tabular}{ll}
\hline Property & Value \\
\hline Radius & $R=1 \mathrm{~m}$ \\
Distance between the two centers ( $x$-axis; $y$-axis) & $x=(2.18 \mathrm{~m} ; 0 \mathrm{~m})$ \\
Initial velocity of the left cylinder $(x$-axis; $y$-axis) & $\dot{x}=(1 \mathrm{~m} / \mathrm{s} ;-0.1 \mathrm{~m} / \mathrm{s})$ \\
Density & $\rho=8.93 \mathrm{~kg} / \mathrm{m}^{3}$ \\
Young's modulus & $E=119.158 \mathrm{~N} / \mathrm{m}^{2}$ \\
Poisson's ratio & $v=0.375$ \\
Yield stress & $\sigma_{0}=10 \mathrm{~N} / \mathrm{m}^{2}$ \\
Hardening parameter & $h=0 \mathrm{~N} / \mathrm{m}^{2}$ \\
Contact penalty & $p_{n}=10^{4}$ \\
\hline
\end{tabular}

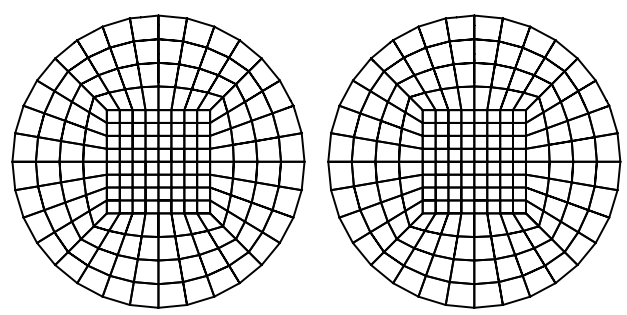

Fig. 4. Mesh of the two cylinders.

cylinder that is initially at rest. The two cylinders are identical (properties reported in Table 2 and mesh illustrated in Fig. 4). The frictionless contact is classically treated for the Newmark algorithm and is treated with the method proposed by Armero and Petöcz (1999) for the conserving algorithm. The simulation occurs in $4 \mathrm{~s}$ and the different time step sizes tested are: 15, 7.5, 3.75 and $1.875 \mathrm{~ms}$. Let us note that for the Newmark algorithm, and the time step size equal to $0.015 \mathrm{~s}$, a penalty parameter of $10^{4}$ does not lead to convergence (it did not lead to convergence in the paper of Meng and Laursen (2002) either). Therefore, a penalty of $10^{3}$ was used in this particular case, and a penalty of $10^{4}$ for the other cases - same values as in Meng and Laursen (2002).

Fig. 5 illustrates deformations and the equivalent plastic strain for the final configurations $(t=4 \mathrm{~s})$. For the EMCA algorithms, the differences between the simulations occurring with different time step sizes, are lower than $5 \%$. This difference is due to the non-smooth contact simulation that allows penetration of the surface. When using the Newmark method, the solution is radically different (little difference in the equivalent plastic strain distribution for $\Delta t=3.75 \mathrm{~ms}$ but more than $100 \%$ error for larger time step sizes). Fig. 6(a) and (b) represent the energy dissipated by plastic deformation. As mentioned previously, if the time step size is increased by a factor of 8 , the Newmark simulation leads to a $100 \%$ different solution. For the same increase, the conserving algorithms with the correcting tensor lead to the same solution while if these tensors are neglected, the solution becomes $0.5 \%$ different. Let us note that our solution is similar with an error of $10 \%$ to the Meng and Laursen (2002) solution, difference resulting from constitutive law (our equivalent yield stress is computed from the Cauchy stress that depends on the compression of the volume). The angular momentum evolution for a time step size equal to $0.015 \mathrm{~s}$ is illustrated in Fig. 7. Only the Newmark solution exhibits some oscillations. The total number of iterations for the Newton-Raphson scheme, reached with a tolerance of $10^{-6}$ (37) for equilibrium, and for the line search system, reached with a tolerance of $10^{-3}$ (36), are reported respectively in Fig. 8(a) and (b). For the largest time step size (15 ms), we can see that the correcting terms allow a reduction of about $25 \%$ in the iterations number of the line 


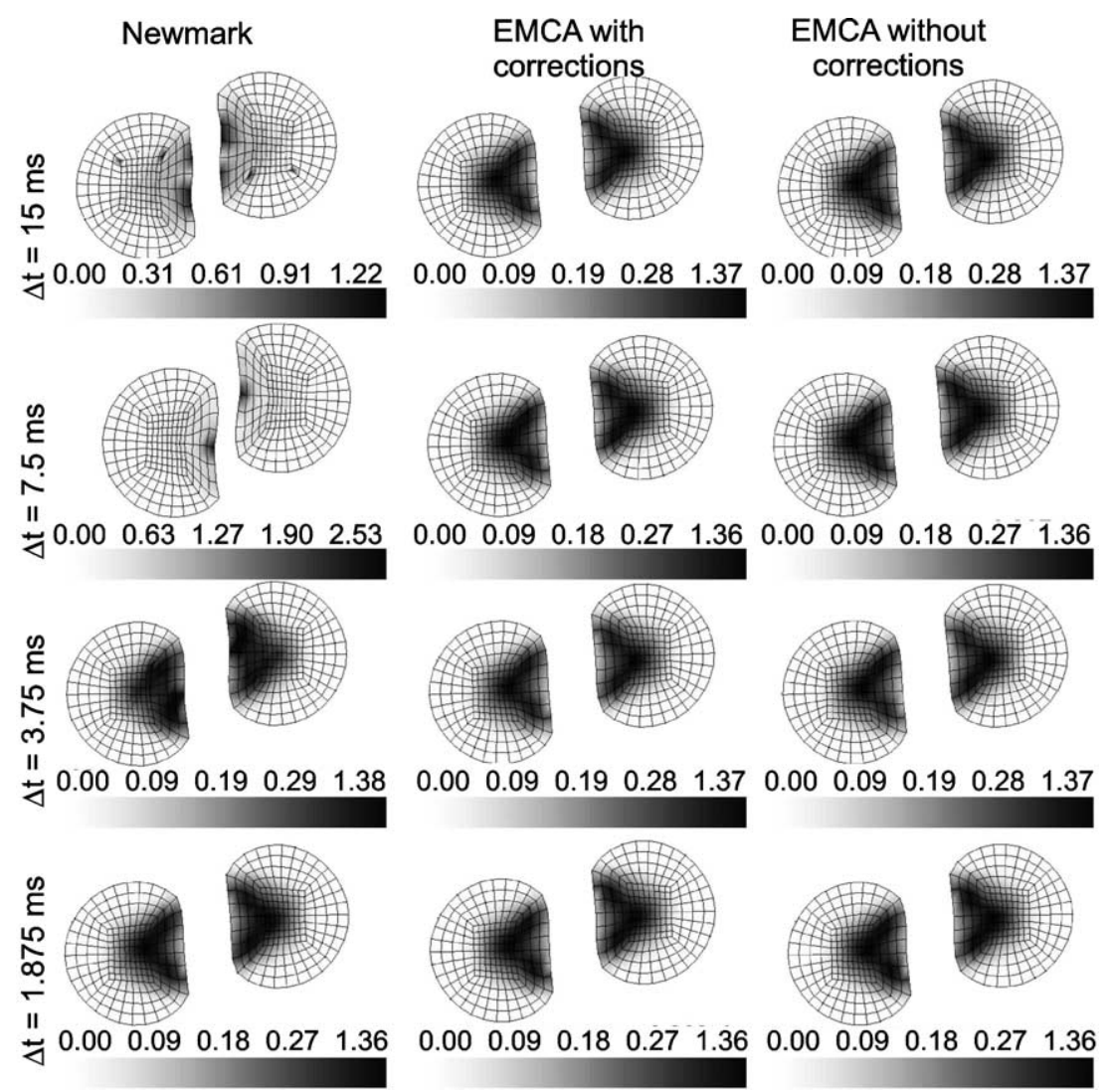

Fig. 5. Final shape and equivalent plastic strain of the two cylinders.

$\diamond-$ EMCA with corrections $(\Delta \mathrm{t}=15 \mathrm{~ms})$

- EMCA without corrections $(\Delta \mathrm{t}=15 \mathrm{~ms})$

$\rightarrow$ Newmark $(\Delta t=15 \mathrm{~ms})$

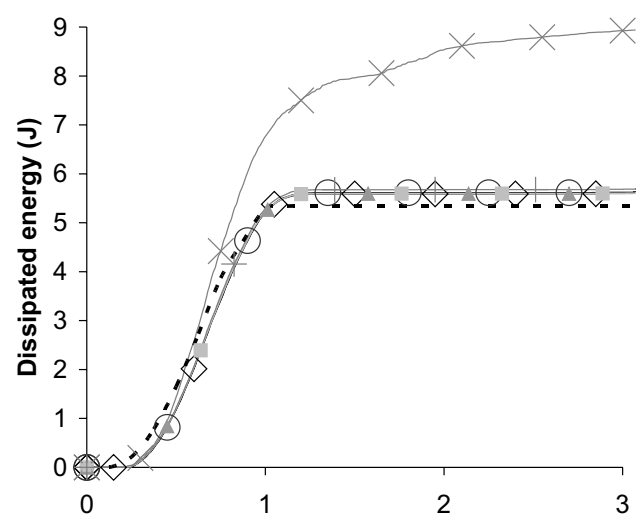

(a)

Time (s) $\rightarrow$ EMCA with corrections $(\Delta \mathrm{t}=1.875 \mathrm{~ms})$

-EMCA without corrections $(\Delta \mathrm{t}=1.875 \mathrm{~ms})$

+ Newmark $(\Delta \mathrm{t}=1.875 \mathrm{~ms})$

- - Meng \& Laursen

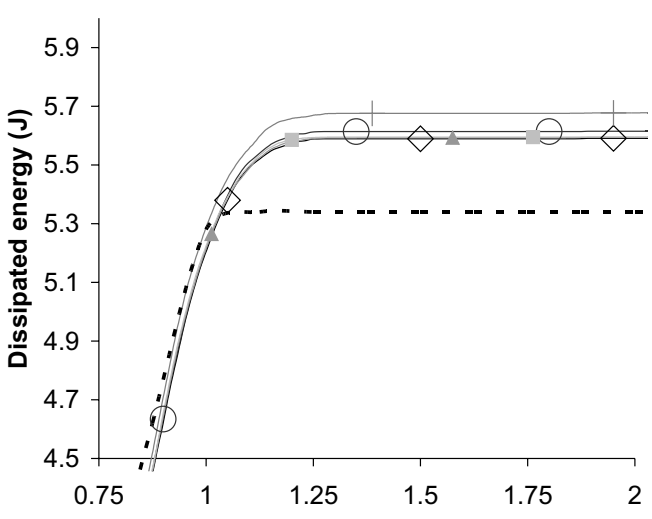

(b)

Fig. 6. Time evolution of the plastic dissipation for the two cylinders: (a) from $t=0 \mathrm{~s}$ to $t=3 \mathrm{~s}$, (b) from $t=0.75 \mathrm{~s}$ to $t=2 \mathrm{~s}$. 


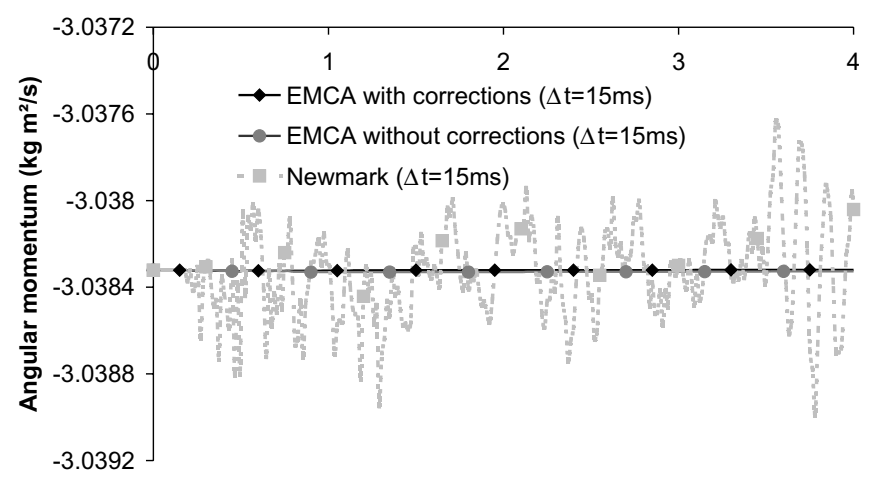

Time (s)

Fig. 7. Time evolution of the angular momentum for the two cylinders ( $\Delta t=15 \mathrm{~ms})$.
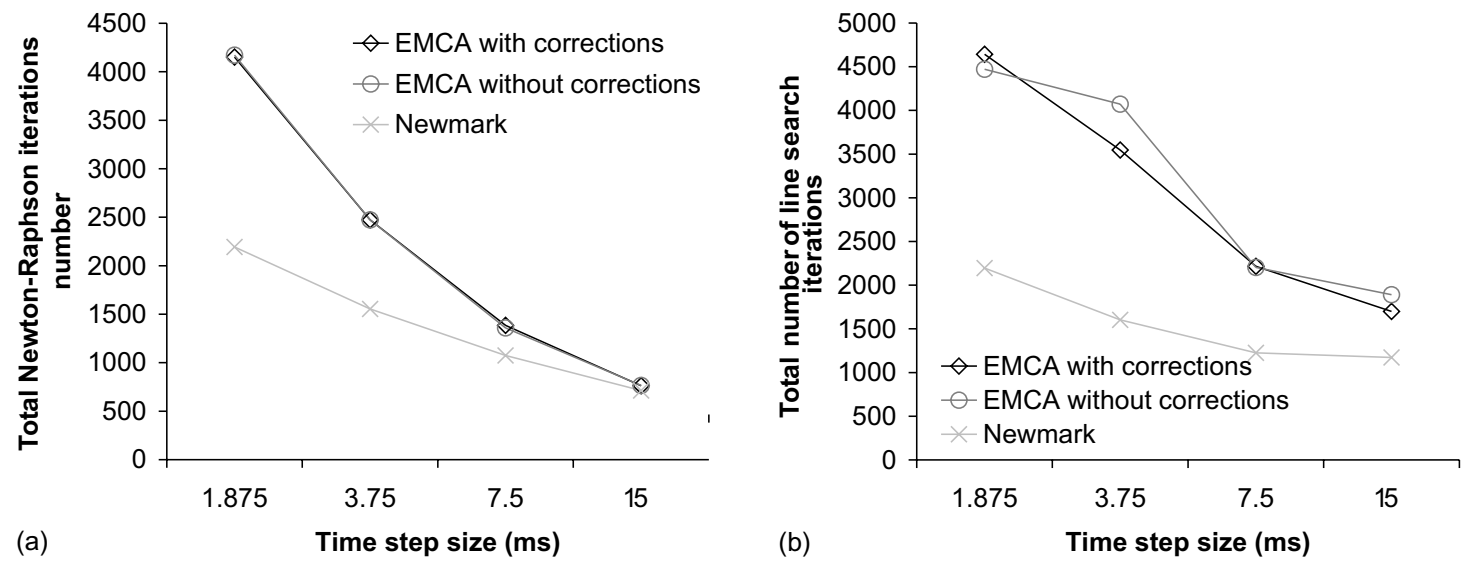

Fig. 8. Iterations number for the two cylinders: (a) Newton-Raphson iterations, (b) line search iterations.

search. The Newmark algorithm is always cheaper than the EMCA algorithms but leads to wrong solutions when the time step size is larger than $1.875 \mathrm{~ms}$.

\subsection{Example 3: the Taylor bar problem}

It consists in a cylindrical bar (Table 3), discretized by 576 elements (Fig. 9). It has an initial velocity $\dot{x}_{0}$. The simulation occurs in $80 \mu$ s and the different time step sizes tested are: $0.5,0.25,0.1 \mu \mathrm{s}$. Fig. 10(a) and (b) illustrate respectively the energy plastically dissipated after $80 \mu \mathrm{s}$ for the different time step sizes, and the error on this energy (related to the simulation with the corrections and a time step equal to $0.1 \mu \mathrm{s}$ ). As in Example 1, when the time step size increases, the energy is overestimated. The use of the EMCA with the correcting tensors allows reducing this overestimation. Fig. 11(a) and (b) illustrates respectively the elastic stored energy obtained from the kinematic energy added to the internal forces work minus the dissipated energy, and the error on this energy. Negative values of the energy, which is absolutely non-physical, are obtained when the time step size increases except for the conserving algorithm using the correcting tensors. This simulation is therefore the only one that leads to consistent results for each time step size. The final 
Table 3

Properties of the Taylor bar problem

\begin{tabular}{ll}
\hline Property & Value \\
\hline External diameter & $d_{\mathrm{e}}=6.4 \mathrm{~mm}$ \\
Length & $l=32.4 \mathrm{~mm}$ \\
Density & $\rho=8930 \mathrm{~kg} / \mathrm{m}^{3}$ \\
Young's modulus & $E=117 \mathrm{E} 9 \mathrm{~N} / \mathrm{m}^{2}$ \\
Poisson's ratio & $v=0.35$ \\
Yield stress & $\sigma_{0}=400 \mathrm{~N} / \mathrm{mm}^{2}$ \\
Hardening parameter & $h=100 \mathrm{~N} / \mathrm{mm}^{2}$ \\
Initial velocity & $\dot{x}_{0}=227 \mathrm{~m} / \mathrm{s}$ \\
\hline
\end{tabular}

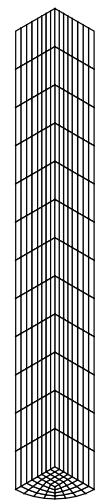

Fig. 9. Discretization of the Taylor bar.
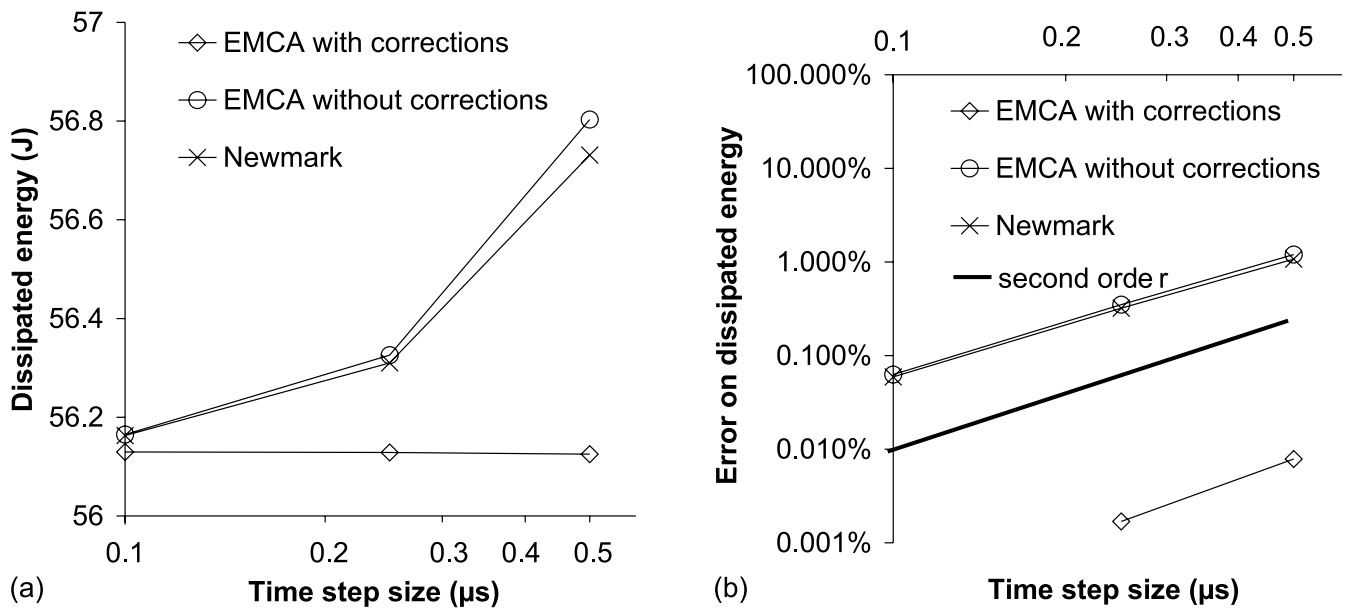

Fig. 10. Final energy (after $80 \mu$ s) for the Taylor bar: (a) dissipated energy, (b) error on the dissipated energy.

total energy is reported in Fig. 12. It appears that the final energy is equal to the initial energy for all schemes. Therefore we can conclude that, since the energy is computed from the work of the internal forces (80), the Newmark algorithm conserves the total energy as demonstrated by Belytschko and Schoeberle (1975) and Hughes (1977). But, for a non-linear system integrated with the Newmark scheme, the work of 


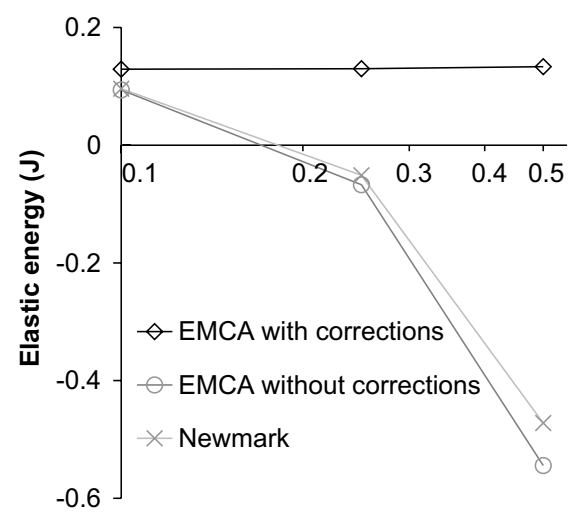

(a)

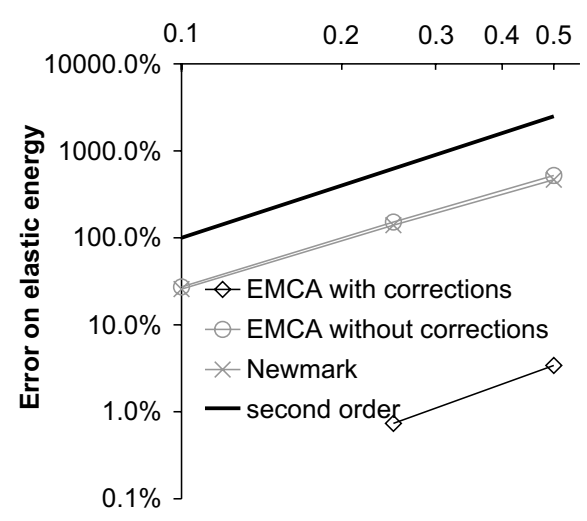

(b)

Time step size ( $\mu \mathrm{s})$

Fig. 11. Final energy (after $80 \mu$ s) for the Taylor bar: (a) elastic energy, (b) error on elastic energy.

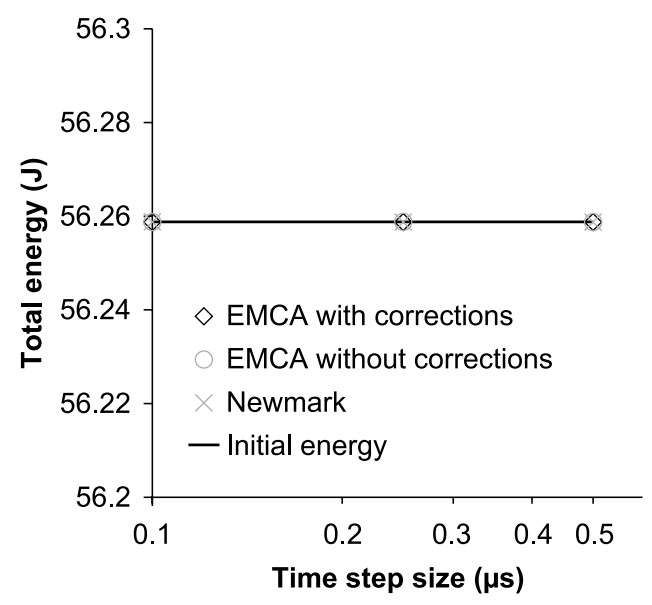

Fig. 12. Final total energy (after $80 \mu$ s) for the Taylor bar.

the internal forces does not correspond to the variation of internal energy added to the plastic dissipation, leading to a repartition of energy not consistent with the physical dynamics. The errors illustrations (Fig. 10 and 11(b)) show that the conserving scheme with the correcting tensors always give the lower error, and that it is second order in the time step size. Fig. 13 illustrates the equivalent plastic strain after $80 \mu$ s. As it has appeared in Fig. 10, the differences between the methods and the time step sizes used are limited (lower than $1 \%$ ).

The total number of iterations for the Newton-Raphson scheme, reached with an equilibrium tolerance of $10^{-8}$ (37), and for the line search system, reached with a tolerance of $10^{-5}(36)$, are respectively illustrated in Fig. 14(a) and (b). When the time step size is increased, the use of the Newmark algorithm and the use of the conserving algorithm without the correcting tensor lead to an increase in the number of iterations. This phenomenon could be explained by the lack of consistency that appears. But, when the correcting tensor are introduced, the total number of iterations is reduced when the time step size increases. For a time step size equal to $0.5 \mu \mathrm{s}$, taking into account these correcting tensors allows reducing the number of iterations of the line search by about $50 \%$ and allows reducing the Newton-Raphson iterations by about $20 \%$. 


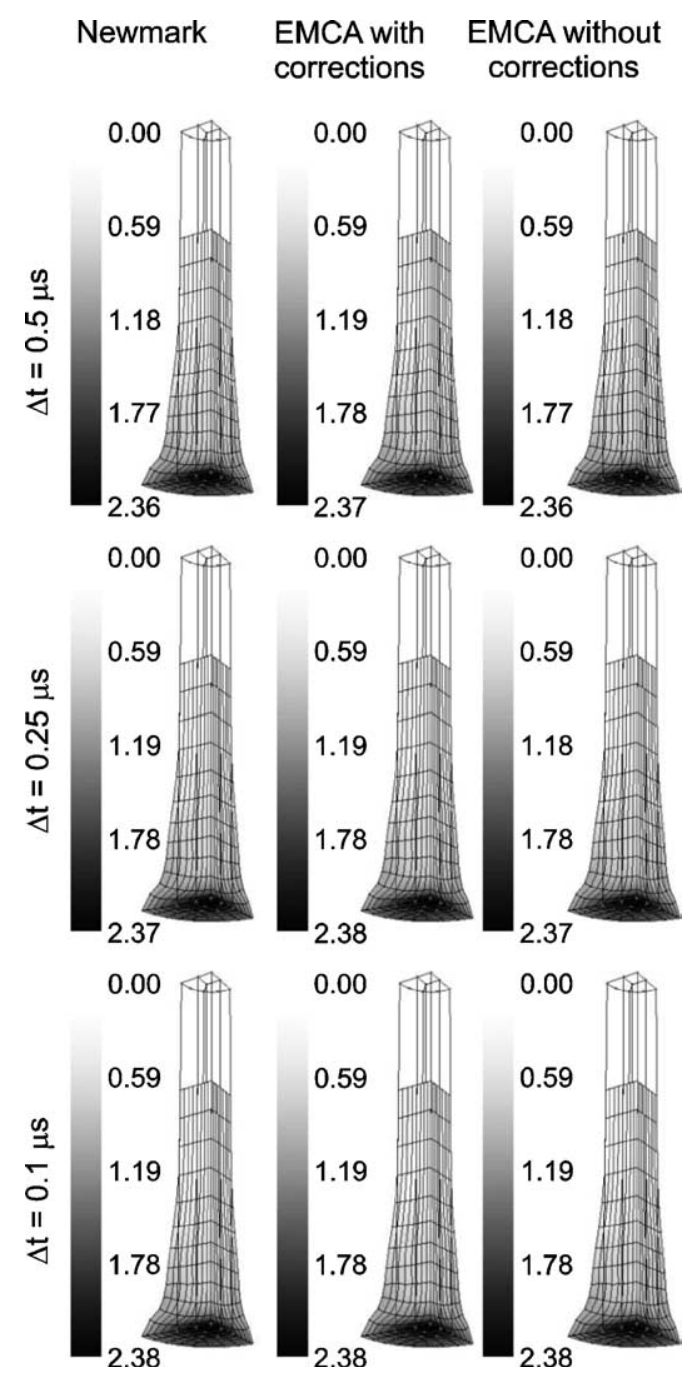

Fig. 13. Final configuration and equivalent plastic strain (after $80 \mu \mathrm{s}$ ) for the Taylor bar.

\section{Conclusions}

We have developed a new formulation of the finite element internal forces associated to the Cauchy stress of an hypoelastic material. With a mid-point scheme, this formulation leads to a thermodynamically consistent dynamics algorithm. The linear and angular momentum are conserved and the energy dissipation corresponds to the physical dissipation resulting from the plastic deformations. In this formulation the correction tensors, that are of the second order in the plastic strain increment and were neglected in our previous work, are now taken into account. When the time step size increases (and the equivalent plastic strain increment increases), neglecting these tensors could lead to a loss of consistency, usually accompanied by an increase in the number of iterations. We have also compared the Newmark solutions with the conserving algorithm ones. It clearly appears that for large time steps, the solutions can diverge, especially 

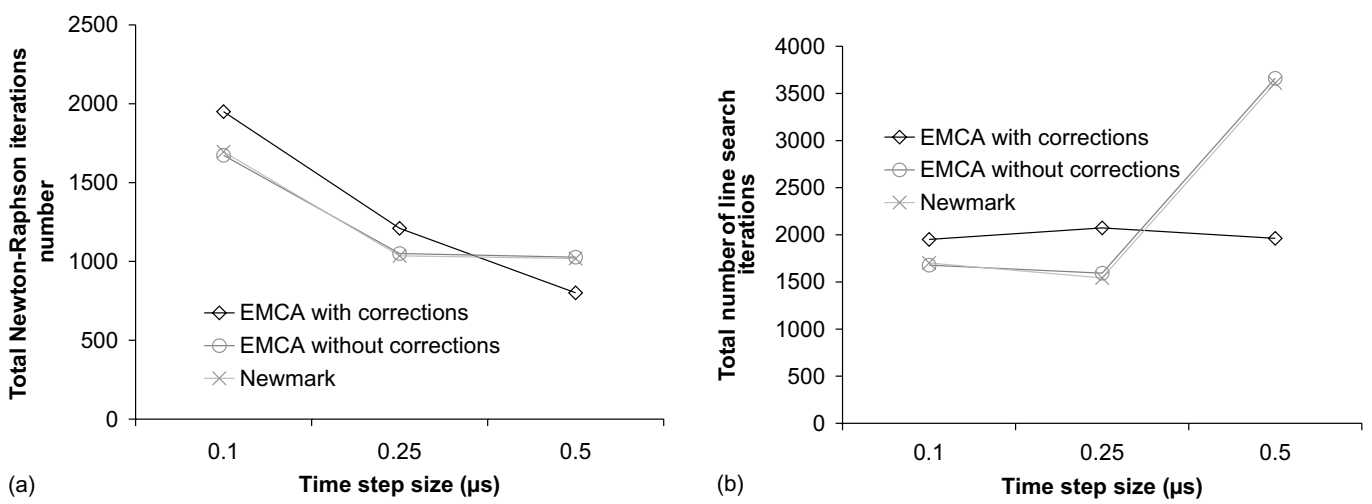

Fig. 14. Iterations number for the Taylor's bar: (a) Newton-Raphson iterations, (b) line search iterations.

when contact interactions occur. Even when no contact occurs, the Newmark algorithm led to a loss of accuracy and an increase of the iterations number when its time step size has been increased. We conclude that the Newmark algorithm conserves the total energy but that the repartition of the energy is not consistent with the physical dynamics in the non-linear range. Therefore, the developed formulation proved to be necessary to integrate non-linear dynamics problems with large time steps.

\section{Acknowledgement}

The authors thank the Belgian National Fund for Scientific Research (FNRS) for its financial support.

\section{Appendix A. A consistent tangent stiffness matrix}

The stiffness matrix defined in (38) will now evaluated for the new expression of the internal forces. First the expression $\vec{F}_{\text {int }}^{*}$ is derived with respect to the positions at time $t_{n+1}$,

$$
\begin{aligned}
\mathbf{K}^{*^{\xi \xi \mu}} & =\frac{\partial\left[\vec{F}_{\text {int }}^{*}\right]^{\xi}}{\partial\left[\vec{x}^{n+1}\right]^{\mu}} \\
& =\frac{1}{2} \int_{V_{0}}\left\{\frac{\partial \mathbf{F}_{n}^{n+1}}{\partial\left[\vec{x}^{n+1}\right]^{\mu}}\left[\Sigma^{n^{\mathrm{T}}} \cdot+\mathbf{C}^{*}\right] \mathbf{f}_{0}^{n^{\mathrm{T}}} \vec{D}^{\xi} J_{0}^{n}\right\} \mathrm{d} V_{0}+\frac{1}{2} \int_{V_{0}}\left\{\left[\mathbf{F}_{n}^{n+1} \cdot+\mathbf{I}\right] \frac{\partial \mathbf{C}^{*}}{\partial\left[\vec{x}^{n+1}\right]^{\mu}} \mathbf{f}_{0}^{n^{\mathrm{T}}} \vec{D}^{\xi} J_{0}^{n}\right\} \mathrm{d} V_{0}
\end{aligned}
$$

With the relations (2) and (4), it leads to

$$
\frac{\partial \mathbf{F}_{n}^{n+1}}{\partial\left[\vec{x}^{n+1}\right]^{\mu}}\left[\Sigma^{n^{\mathrm{T}}}+\mathbf{C}^{*}\right]=\mathscr{N}^{1} \bullet\left[\vec{B}^{n}\right]^{\mu}
$$

with first order tensor $\left[\vec{B}^{n}\right]^{\mu}$ defined by $\left[\vec{B}^{n}\right]^{\mu}=\mathbf{f}_{0}^{n^{\mathrm{T}}} \vec{D}^{\mu}$, with fourth order tensor $\mathscr{N}^{1}$ defined by $\mathscr{N}_{i j k l}^{1}=$ $\mathbf{I}_{i k}\left[\boldsymbol{\Sigma}_{l j}^{n}+\mathbf{C}_{j l}^{*}\right]$, and with operation $\left[\vec{B}^{n}\right]^{\xi} \bullet \mathscr{N}^{1} \bullet\left[\vec{B}^{n}\right]^{\mu}$ defined by

$$
\left[\left[\vec{B}^{n}\right]^{\xi} \bullet \mathscr{N}^{1} \bullet\left[\vec{B}^{n}\right]^{\mu}\right]_{i k}=\left[\vec{B}^{n}\right]_{j}^{\xi} \mathscr{N}_{i j k l}^{1}\left[\vec{B}^{n}\right]_{l}^{\mu}
$$


Now let us study the derivation of $\mathbf{C}^{*}$. We can write

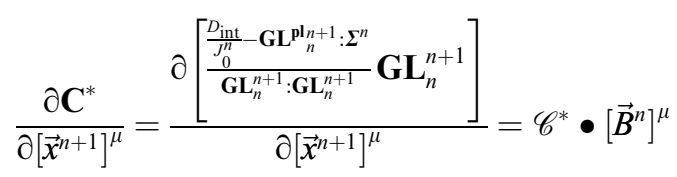

Let us evaluate $\mathscr{C}^{*}$. With the relations (2) and (4), it leads to

$$
\frac{\partial \mathbf{G L}_{n}^{n+1}}{\partial\left[\vec{x}^{n+1}\right]^{\mu}}=\mathscr{C}^{*^{1}} \bullet\left[\vec{B}^{n}\right]^{\mu}
$$

with $\mathscr{C}_{i j k l}^{*}=\frac{1}{2}\left[\delta_{i l}\left[\mathbf{F}_{n}^{n+1}\right]_{k j}+\delta_{j l}\left[\mathbf{F}_{n}^{n+1}\right]_{k i}\right]$. Using this results yields

$$
\frac{\partial\left[\frac{1}{\mathbf{G L}_{n}^{n+1}: \mathbf{G L}_{n}^{n+1}}\right]}{\partial\left[\vec{x}^{n+1}\right]^{\mu}} \mathbf{G L}_{n}^{n+1}=\mathscr{C}^{*^{2}} \bullet\left[\vec{B}^{n}\right]^{\mu}
$$

with $\mathscr{C}_{i j k l}^{*^{2}}=-\frac{2}{\left[\mathbf{G L}_{n}^{n+1}: \mathbf{G L}_{n}^{n+1}\right]^{2}} \mathbf{G L}_{i j}\left[\mathbf{F}_{n}^{n+1} \mathbf{G L}_{n}^{n+1}\right]_{k l}$.

Now let us assume that the derivation of the internal dissipation (that is material dependent and that will be computed in Appendix B) can take the following formulation:

$$
\frac{\partial D_{\text {int }}}{\partial\left[\vec{x}^{n+1}\right]^{\mu}}=\mathbf{D}_{\text {int }}\left[\vec{B}^{n+1}\right]^{\mu}=\mathbf{D}_{\text {int }} \mathbf{f}_{n}^{n+1^{\mathrm{T}}}\left[\vec{B}^{n}\right]^{\mu}
$$

Therefore, it leads to

$$
\frac{\partial D_{\text {int }}}{\partial\left[\vec{x}^{n+1}\right]^{\mu}} \mathbf{G L}_{n}^{n+1}=\mathscr{C}^{*^{3}} \bullet\left[\vec{B}^{n}\right]^{\mu}
$$

with $\mathscr{C}_{i j k l}^{*^{3}}=\mathbf{G L}_{i j}\left[\mathbf{D}_{\text {int }} \mathbf{f}_{n}^{n+1^{\mathrm{T}}}\right]_{k l}$.

To be able to derive the term $\mathbf{G L}_{n}^{\mathbf{p}_{n+1}}$, it is decomposed into $\mathbf{G L}_{n}^{n+1}-\mathbf{G L}_{n}^{\mathbf{e}_{n+1}}$. Using (A.5) and the symmetric property of $\Sigma$, the $\mathbf{G L}_{n}^{n+1}$ part becomes

$$
-\left[\frac{\partial \mathbf{G L}_{n}^{n+1}}{\partial\left[\vec{x}^{n+1}\right]^{\mu}}: \Sigma^{n}\right] \mathbf{G L}_{n}^{n+1}=\mathscr{C}^{*^{4}} \bullet\left[\vec{B}^{n}\right]^{\mu}
$$

with $\mathscr{C}_{i j k l}^{*^{4}}=-\mathbf{G L}_{n}^{n+1} i j\left[\mathbf{F}_{n}^{n+1} \Sigma^{n}\right]_{k l}$. The $\mathbf{G L}_{n}^{\mathbf{e l}_{n+1}}$ part is obtained using the definitions (62)-(64). With the definition of the Hooke tensor (15), the definition of the plastic correction (18) and using the fact that for $J 2$ plasticity the trace of $\mathbf{N}^{\mathbf{c}}$ is equal to zero, it comes

$$
\begin{aligned}
& \mathbf{E}_{n}^{\mathbf{e} n+1}=\mathbf{E}_{n}^{n+1}-\gamma \mathbf{N}^{\mathbf{c}} \\
& \frac{1}{2} \ln \left[2 \mathbf{G} \mathbf{L}_{n}^{\mathbf{e}(n+1}+\mathbf{I}\right]=\frac{1}{2} \ln \left[2 \mathbf{G L}_{n}^{n+1}+\mathbf{I}\right]-\gamma \mathbf{N}^{\mathbf{c}} \\
& \mathbf{G L}{ }_{n}^{\mathbf{e} \mathbf{l} n+1}=\frac{1}{2} \exp \left\{\ln \left[2 \mathbf{G L}_{n}^{n+1}+\mathbf{I}\right]-2 \gamma \mathbf{N}^{\mathbf{c}}\right\}-\frac{1}{2} \mathbf{I}
\end{aligned}
$$

Assuming the norm of any four order tensor $\mathbf{M}$ remains close to zero, derivation of the exponential of $\mathbf{M}$ can be approximated by

$$
\partial \exp \mathbf{M} \simeq \frac{1}{2}[\partial \mathbf{M} \exp \mathbf{M}+\exp \mathbf{M} \partial \mathbf{M}]
$$

Therefore, it yields

$$
\begin{aligned}
2 \frac{\partial\left[\mathbf{G L}_{n}^{\mathbf{e l}} n+1\right.}{\partial\left[\vec{x}^{n+1}\right]^{\mu}}= & \frac{\partial\left[\ln \left[2 \mathbf{G L}_{n}^{n+1}+\mathbf{I}\right]-2 \gamma \mathbf{N}^{\mathbf{c}}\right]_{i m}}{\partial\left[\vec{x}^{n+1}\right]^{\mu}}\left[2 \mathbf{L}_{n}^{\mathbf{e l} n+1}+\mathbf{I}\right]_{m j} \\
& +\left[2 \mathbf{G L}_{n}^{\mathbf{e} \mathbf{e}_{n+1}}+\mathbf{I}\right]_{i m} \frac{\partial\left[\ln \left[2 \mathbf{G} \mathbf{L}_{n}^{n+1}+\mathbf{I}\right]-2 \gamma \mathbf{N}^{\mathbf{c}}\right]_{m j}}{\partial\left[\vec{x}^{n+1}\right]^{\mu}}
\end{aligned}
$$


Let $\overline{\mathscr{M}}$ be the forth order tensor defined by

$$
\mathbf{R}_{n}^{n+1} \frac{\partial\left[\mathscr{H}: \mathbf{E}_{n}^{n+1}-2 g \gamma \mathbf{N}^{\mathrm{c}}\right]}{\partial\left[\vec{x}^{n+1}\right]^{\mu}} \mathbf{R}_{n}^{n+1^{\mathrm{T}}}=\overline{\mathscr{M}} \bullet\left[\vec{B}^{n+1}\right]^{\mu}=\overline{\mathscr{M}} \bullet \mathbf{f}_{n}^{n+1^{\mathrm{T}}}\left[\vec{B}^{n}\right]^{\mu}
$$

the expression of this tensor will computed in Appendix B. Using this definition, the relation (A.12) can be rewritten as

$$
\begin{aligned}
& 2 \frac{\partial\left[\mathbf{G L}_{n}^{\mathbf{e l} n+1}\right]_{i j}}{\partial\left[\vec{x}^{n+1}\right]_{k}^{\mu}}=\left[\mathbf{R}_{n}^{n+1}\right]_{p i}\left[\mathscr{H}^{-1} \overline{\mathscr{M}}\right]_{p q k n}\left[\mathbf{R}_{n}^{n+1}\right]_{q m}\left[2 \mathbf{G L}_{n}^{\text {el }} n+1+\mathbf{I}\right]_{m j}\left[\mathbf{f}_{n}^{n+1}\right]_{l n}\left[\vec{B}^{n}\right]_{l}^{\mu} \\
& +\left[2 \mathbf{G L}_{n}^{\mathbf{e} \mathbf{l} n+1}+\mathbf{I}\right]_{i m}\left[\mathbf{R}_{n}^{n+1}\right]_{p m}\left[\mathscr{H}^{-1} \overline{\mathscr{M}}\right]_{p q k n}\left[\mathbf{R}_{n}^{n+1}\right]_{q j}\left[\mathbf{f}_{n}^{n+1}\right]_{l n}\left[\vec{B}^{n}\right]_{l}^{\mu}
\end{aligned}
$$

Let $\mathbf{C}^{5}$ be the matrix defined by $\mathbf{R} \Sigma^{n} \mathbf{U}^{\mathrm{el}}{ }^{2} \mathbf{R}^{\mathrm{T}}$. Using the symmetric properties of $\Sigma$, of $\mathbf{U}^{\mathrm{el}}$ and the symmetric properties $\left(\left[\mathscr{H}^{-1} \overline{\mathscr{M}}\right]_{i j k l}=\left[\mathscr{H}^{-1} \overline{\mathscr{M}}\right]_{j i k l}\right)$ of the material tensor, relation (A.14) yields

$$
\left[\frac{\partial \mathbf{G L}_{n}^{\mathbf{e l}_{n+1}}}{\partial\left[\vec{x}^{n+1}\right]^{\mu}}: \Sigma^{n}\right] \mathbf{G L}_{n}^{n+1}=\mathscr{C}^{*^{5}} \bullet\left[\vec{B}^{n}\right]^{\mu}
$$

with $\mathscr{C}_{i j k l}^{*^{5}}=\mathbf{G L}_{n}^{n+1} i j\left\{\mathbf{C}^{5}:\left[\mathscr{H}^{-1} \overline{\mathscr{M}}\right]\right\}_{k n}\left[\mathbf{f}_{n}^{n+1}\right]_{l n}$, where $[\mathbf{M}: \mathscr{H}]_{k l}$ is equal to $\mathbf{M}_{i j} \mathscr{H}_{i j k l}$. Finally, tensor $\mathscr{C}^{*}$ defined in relation (A.4) can be written, using relations (A.5), (A.6), (A.8), (A.9) and (A.15) as

$$
\begin{aligned}
\mathscr{C}^{*}= & {\left[\frac{\frac{D_{\text {int }}}{J_{0}^{n}}-\mathbf{G L}_{n}^{\mathbf{p l}_{n+1}}: \boldsymbol{\Sigma}^{n}}{\mathbf{G L}_{n}^{n+1}: \mathbf{G L}_{n}^{n+1}}\right] \mathscr{C}^{*^{1}}+\left[\frac{D_{\mathrm{int}}}{J_{0}^{n}}-\mathbf{G L}_{n}^{\mathbf{p} \mathbf{l} n+1}: \Sigma^{n}\right] \mathscr{C}^{*^{2}}+\left[\frac{1}{J_{0}^{n} \mathbf{G L}_{n}^{n+1}: \mathbf{G L}_{n}^{n+1}}\right] \mathscr{C}^{*^{3}} } \\
& +\left[\frac{1}{\mathbf{G L}_{n}^{n+1}: \mathbf{G L}_{n}^{n+1}}\right] \mathscr{C}^{*^{4}}+\left[\frac{1}{\mathbf{G L}_{n}^{n+1}: \mathbf{G L}_{n}^{n+1}}\right] \mathscr{C}^{*^{5}}
\end{aligned}
$$

Therefore, using the relations (A.2) and (A.4), the expression (A.1) becomes

$$
\mathbf{K}^{*^{\xi \mu}}=\frac{1}{2} \int_{V_{0}}\left\{\left[\vec{B}^{n}\right]^{\xi} \bullet \mathscr{K}^{*} \bullet\left[\vec{B}^{n}\right]^{\mu} J_{0}^{n}\right\} \mathrm{d} V_{0}
$$

with $\mathscr{K}_{i j k l}^{*}$ defined by $\mathscr{N}_{i j k l}^{1}+\left[\mathbf{F}_{n}^{n+1}+\mathbf{I}\right]_{i m} \mathscr{C}_{m j k l}^{*}$.

Now the expression $\vec{F}_{\text {int }}^{* *}$ is derived with respect to the positions at time $t_{n+1}$,

$$
\begin{aligned}
\mathbf{K}^{* * \xi \mu}= & \frac{\partial\left[\vec{F}_{\text {int }}^{* *}\right]^{\xi}}{\partial\left[\vec{x}^{n+1}\right]^{\mu}} \\
= & \frac{1}{2} \int_{V_{0}}\left\{\frac{\partial \mathbf{f}_{n}^{n+1}}{\partial\left[\vec{x}^{n+1}\right]^{\mu}}\left[\boldsymbol{\Sigma}^{n+1^{\mathrm{T}}}+\mathbf{C}^{* *}\right] \mathbf{f}_{0}^{n+1^{\mathrm{T}}} \vec{D}^{\xi} J_{0}^{n+1}\right\} \mathrm{d} V_{0}+\frac{1}{2} \int_{V_{0}}\left\{\left[\mathbf{f}_{n}^{n+1}+\mathbf{I}\right] \frac{\partial \boldsymbol{\Sigma}^{\mathbf{n}+\mathbf{1}^{\mathrm{T}}}}{\partial\left[\vec{x}^{n+1}\right]^{\mu}} \mathbf{f}_{0}^{n+1^{\mathrm{T}}} \vec{D}^{\xi} J_{0}^{n+1}\right\} \mathrm{d} V_{0} \\
& +\frac{1}{2} \int_{V_{0}}\left\{\left[\mathbf{f}_{n}^{n+1}+\mathbf{I}\right] \frac{\partial \mathbf{C}^{* *}}{\partial\left[\vec{x}^{n+1}\right]^{\mu}} \mathbf{f}_{0}^{n+1^{\mathrm{T}}} \vec{D}^{\xi} J_{0}^{n+1}\right\} \mathrm{d} V_{0}+\frac{1}{2} \int_{V_{0}}\left\{\left[\mathbf{f}_{n}^{n+1}+\mathbf{I}\right]\left[\boldsymbol{\Sigma}^{n+1^{\mathrm{T}}}+\mathbf{C}^{* *}\right] \frac{\partial\left[\mathbf{f}_{0}^{n+1^{\mathrm{T}}} \vec{D}^{\xi} J_{0}^{n+1}\right]}{\partial\left[\left[^{n+1}\right]^{\mu}\right.}\right\} \mathrm{d} V_{0}
\end{aligned}
$$

The first part of relation (A.18) is evaluated. With relation $\partial \mathbf{f}=-\mathbf{f} \partial \mathbf{F f}$ and using relations (2) and (4), it leads to

$$
\frac{\partial \mathbf{f}_{n}^{n+1}}{\partial\left[\vec{x}^{n+1}\right]^{\mu}}\left[\boldsymbol{\Sigma}^{n+1^{\mathrm{T}}}+\mathbf{C}^{* *}\right]=-\mathbf{F}_{0}^{n} \mathbf{f}_{0}^{n+1} \frac{\partial \mathbf{F}_{0}^{n+1}}{\partial\left[\vec{x}^{n+1}\right]^{\mu}} \mathbf{f}_{0}^{n+1}\left[\boldsymbol{\Sigma}^{n+1^{\mathrm{T}}}+\mathbf{C}^{* *}\right]=\mathscr{N}^{2} \bullet\left[\vec{B}^{n+1}\right]^{\mu}
$$

with $\mathscr{N}_{i j k l}^{2}=-\left[\mathbf{f}_{n}^{n+1}\right]_{i k}\left[\Sigma_{l j}^{n+1}+\mathbf{C}_{j l}^{* *}\right]$. The second part of the relation (A.18) is evaluated through the derivation of $\Sigma^{n+1}$, 


$$
\frac{\partial \boldsymbol{\Sigma}^{n+1}}{\partial\left[\vec{x}^{n+1}\right]^{\mu}}=\frac{\partial\left[\mathbf{R}_{n}^{n+1} \boldsymbol{\Sigma}^{\mathbf{c}^{n+1}} \mathbf{R}_{n}^{n+1^{\mathrm{T}}}\right]}{\partial\left[\vec{x}^{n+1}\right]^{\mu}}=\mathbf{R}_{n}^{n+1} \frac{\partial \boldsymbol{\Sigma}^{\mathbf{c}^{n+1}}}{\partial\left[\vec{x}^{n+1}\right]^{\mu}} \mathbf{R}_{n}^{n+1^{\mathrm{T}}}+\frac{\partial \mathbf{R}_{n}^{n+1}}{\partial\left[\vec{x}^{n+1}\right]^{\mu}} \mathbf{R}_{n}^{n+1^{\mathrm{T}}} \boldsymbol{\Sigma}^{n+1}+\boldsymbol{\Sigma}^{n+1} \mathbf{R}_{n}^{n+1} \frac{\partial \mathbf{R}_{n}^{n+1^{\mathrm{T}}}}{\partial\left[\vec{x}^{n+1}\right]^{\mu}}
$$

Using definition of $\overline{\mathscr{M}}$ in relation (A.13), it leads to

$$
\mathbf{R}_{n}^{n+1} \frac{\partial \boldsymbol{\Sigma}^{\mathbf{c}^{n+1}}}{\partial\left[\vec{x}^{n+1}\right]^{\mu}} \mathbf{R}_{n}^{n+1^{\mathrm{T}}}=\mathbf{R}_{n}^{n+1} \frac{\partial\left[\mathscr{H}: \mathbf{E}_{n}^{n+1}-2 g \gamma \mathbf{N}^{\mathrm{c}}\right]}{\partial\left[\vec{x}^{n+1}\right]^{\mu}} \mathbf{R}_{n}^{n+1^{\mathrm{T}}}=\overline{\mathscr{M}} \bullet\left[\vec{B}^{n+1}\right]^{\mu}
$$

Moreover, we have (Ponthot, 1995; Nagtegaal and Veldpaus, 1984)

$$
\begin{gathered}
\frac{\partial \mathbf{R}_{n}^{n+1}}{\partial\left[\vec{x}^{n+1}\right]^{\mu}} \mathbf{R}_{n}^{n+1^{\mathrm{T}}} \simeq \mathscr{J}^{1} \bullet\left[\vec{B}^{n+1}\right]^{\mu} \\
\mathbf{R}_{n}^{n+1} \frac{\partial \mathbf{R}_{n}^{n+1^{\mathrm{T}}}}{\partial\left[\vec{x}^{n+1}\right]^{\mu}} \simeq-\mathscr{J}^{1} \bullet\left[\vec{B}^{n+1}\right]^{\mu}
\end{gathered}
$$

with $\mathscr{J}^{1}=\frac{1}{2}\left[\delta_{i k} \delta_{j l}-\delta_{i l} \delta_{j k}\right]$. Therefore, if we denote by $\mathscr{J}(\mathbf{M})$ the Jaumann four order tensor such that

$$
[\mathscr{J}(\mathbf{M})]_{i j k l}=\frac{1}{2}\left[\mathbf{M}_{i l} \delta_{j k}-\mathbf{M}_{i k} \delta_{j l}+\mathbf{M}_{j l} \delta_{i k}-\mathbf{M}_{k j} \delta_{i l}\right]
$$

the relation (A.20) can be rewritten, using relations (A.21) and (A.22) as

$$
\frac{\partial \boldsymbol{\Sigma}^{n+1}}{\partial\left[\vec{x}^{n+1}\right]^{\mu}}=\left[\overline{\mathscr{M}}+\mathscr{J}\left(\boldsymbol{\Sigma}^{n+1}\right)\right] \bullet\left[\vec{B}^{n+1}\right]^{\mu}
$$

Let us note that for the implementation described in Section 3.3, this term is decomposed into a tensor relative to the volumic point (pressure) and into a tensor relative to the deviatoric points (deviatoric stress). So the $\vec{B}$ vectors depend on the Gauss point at which the values are computed. Now the third part of relation (A.18) is developed. We can write

$$
\frac{\partial \mathbf{C}^{* *}}{\partial\left[\vec{x}^{n+1}\right]^{\mu}}=\frac{\partial\left[\frac{D_{\text {int }}-\mathbf{A}_{n}^{\mathrm{pl} n+1}: \Sigma^{n+1}}{\mathbf{A}_{n}^{n+1}: \mathbf{A}_{n}^{n+1}} \mathbf{A}_{n}^{n+1}\right]}{\partial\left[\vec{x}^{n+1}\right]^{\mu}}=\mathscr{C}^{* *} \bullet\left[\vec{B}^{n+1}\right]^{\mu}
$$

Let us evaluate $\mathscr{C}^{* *}$. With the relations (2) and (4), it leads to

$$
\frac{\partial \mathbf{A}_{n}^{n+1}}{\partial\left[\vec{x}^{n+1}\right]^{\mu}}=\mathscr{C}^{* *^{1}} \bullet\left[\vec{B}^{n+1}\right]^{\mu}
$$

with $\mathscr{C}_{i j k l}^{* * 1}=\frac{1}{2}\left[\delta_{i l} \delta_{j k}+\delta_{i k} \delta_{j l}-2 \delta_{i l} \mathbf{A}_{j k}-2 \delta_{j l} \mathbf{A}_{i k}\right]$. Using this results yields

$$
\frac{\partial\left[\frac{1}{\mathbf{A}_{n}^{n+1}: \mathbf{A}_{n}^{n+1}}\right]}{\partial\left[\vec{x}^{n+1}\right]^{\mu}} \mathbf{A}_{n}^{n+1}=\mathscr{C}^{* * 2} \bullet\left[\vec{B}^{n+1}\right]^{\mu}
$$

with $\mathscr{C}_{i j k l}^{* *} 2=-\frac{2}{\left[\mathbf{A}_{n}^{n+1}: \mathbf{A}_{n}^{n+1}\right]^{2}} \mathbf{A}_{i j}\left[\mathbf{A}-2 \mathbf{A}^{2}\right]_{k l}$. Using the definition of $\mathbf{D}_{\text {int }}$ (A.7), it leads to

$$
\frac{\partial D_{\text {int }}}{\partial\left[\vec{x}^{n+1}\right]^{\mu}} \mathbf{A}_{n}^{n+1}=\mathscr{C}^{* * 3} \bullet\left[\vec{B}^{n+1}\right]^{\mu}
$$

with $\mathscr{C}_{i j k l}^{* *^{3}}=\mathbf{A}_{i j} \mathbf{D}_{\text {int }} k l$. Moreover, we have

$$
\frac{\frac{1}{J_{0}^{n+1}}}{\partial\left[\vec{x}^{n+1}\right]^{\mu}} \mathbf{A}_{n}^{n+1}=\mathscr{C}^{* * 4} \bullet\left[\vec{B}^{n+1}\right]^{\mu}
$$


with $\mathscr{C}_{i j k l}^{* * 4}=-\frac{1}{J^{n+1}} \mathbf{A}_{i j} \delta_{k l}$. To be able to derive the term $\mathbf{A}_{n}^{\mathbf{p l} \mathbf{l}_{n+1}}$, it is decomposed in $\mathbf{A}_{n}^{n+1}-\mathbf{A}_{n}^{\mathbf{e} \mathbf{l}_{n+1}}$. Using (A.26) and the symmetric properties of $\Sigma$, the $\mathbf{A}_{n}^{n+1}$ part becomes

$$
-\left[\frac{\partial \mathbf{A}_{n}^{n+1}}{\partial\left[\vec{x}^{n+1}\right]^{\mu}}: \Sigma^{n+1}\right] \mathbf{A}_{n}^{n+1}=\mathscr{C}^{* * 5} \bullet\left[\vec{B}^{n+1}\right]^{\mu}
$$

with $\mathscr{C}_{i j k l}^{* *^{5}}=-\mathbf{A}_{n}^{n+1} i j\left[\Sigma^{n+1}-2 \mathbf{A} \Sigma^{n+1}\right]_{k l}$ (using the symmetric properties of $\boldsymbol{\Sigma}$ ). The $\mathbf{A}_{n}^{\mathbf{e l}_{n+1}}$ part is obtained using the definitions (62)-(64). With the definition of $\mathscr{J}(\mathbf{M})$ in relation (A.23), it leads

$$
\begin{aligned}
& \frac{\partial \mathbf{A}_{n}^{\mathbf{e}^{n+1}}}{\partial\left[\vec{x}^{n+1}\right]^{\mu}}=-\frac{1}{2} \frac{\partial \mathbf{R}_{n}^{n+1} \mathbf{U}_{n}^{\mathbf{e}^{n+1}-2} \mathbf{R}_{n}^{n+1^{\mathrm{T}}}}{\partial\left[\vec{x}^{n+1}\right]^{\mu}}=\mathscr{J}\left(\mathbf{A}^{\mathbf{e l}}\right) \bullet\left[\vec{B}^{n+1}\right]^{\mu}-\frac{1}{2} \mathbf{R}_{n}^{n+1} \frac{\partial \mathbf{U}_{n}^{\mathbf{e}^{n+1}-2}}{\partial\left[\vec{x}^{n+1}\right]^{\mu}} \mathbf{R}_{n}^{n+1^{\mathrm{T}}} \\
& =\mathscr{J}\left(\mathbf{A}^{\mathbf{e}}\right) \bullet\left[\vec{B}^{n+1}\right]^{\mu}+\frac{1}{2} \mathbf{R}_{n}^{n+1} \mathbf{U}_{n}^{\mathbf{e}^{n+1^{-2}}} \frac{\partial \mathbf{U}_{n}^{\mathbf{e} \mathbf{l} n+1^{2}}}{\partial\left[\vec{x}^{n+1}\right]^{\mu}} \mathbf{U}_{n}^{\mathbf{e}^{n+1^{-2}}} \mathbf{R}_{n}^{n+1^{\mathrm{T}}} \\
& =\mathscr{J}\left(\mathbf{A}^{\mathbf{e l}}\right) \bullet\left[\vec{B}^{n+1}\right]^{\mu}+\mathbf{R}_{n}^{n+1} \mathbf{U}_{n}^{\mathrm{e}^{n+1^{-2}}} \frac{\partial \mathbf{G} \mathbf{L}_{n}^{\mathbf{e} \mathbf{l}_{n+1}}}{\partial\left[\vec{x}^{n+1}\right]^{\mu}} \mathbf{U}_{n}^{\mathrm{e}^{n+1^{-2}}} \mathbf{R}_{n}^{n+1^{\mathrm{T}}}
\end{aligned}
$$

The relation (A.14) can be rewritten as

$$
\frac{\partial\left[\mathbf{G L}_{n}^{\left.\mathrm{e}\right|^{n+1}}\right]}{\partial\left[\vec{x}^{n+1}\right]^{\mu}}=\frac{1}{2} \mathbf{R}_{n}^{n+1^{\mathrm{T}}}\left[\mathscr{H}^{-1} \overline{\mathscr{M}}\right] \mathbf{R}_{n}^{n+1} \mathbf{U}_{n}^{\mathrm{e}^{n+1^{2}}} \bullet\left[\vec{B}^{n+1}\right]^{\mu}+\frac{1}{2} \mathbf{U}_{n}^{\mathrm{e}^{n+11^{2}}} \mathbf{R}_{n}^{n+1^{\mathrm{T}}}\left[\mathscr{H}^{-1} \overline{\mathscr{M}}\right] \mathbf{R}_{n}^{n+1} \bullet\left[\vec{B}^{n+1}\right]^{\mu}
$$

Combining relation (A.31) and (A.32) yields

$$
\frac{\partial \mathbf{A}_{n}^{\left.\mathbf{e}\right|^{n+1}}}{\partial\left[\vec{x}^{n+1}\right]^{\mu}}=\mathscr{J}\left(\mathbf{A}^{\mathbf{e l}}\right) \bullet\left[\vec{B}^{n+1}\right]^{\mu}+\left[\mathscr{H}^{-1} \overline{\mathscr{M}}\right] \bullet\left[\vec{B}^{n+1}\right]^{\mu}-\left[\mathbf{A}^{\mathbf{e l}}\left[\mathscr{H}^{-1} \overline{\mathscr{M}}\right]+\left[\mathscr{H}^{-1} \overline{\mathscr{M}}\right] \mathbf{A}^{\mathbf{e l}}\right] \bullet\left[\vec{B}^{n+1}\right]^{\mu}
$$

Therefore, defining

$$
\mathbf{C}_{i j}^{* * 6}=\boldsymbol{\Sigma}_{m n}^{n+1} \mathscr{J}\left(\mathbf{A}^{\mathrm{el}}\right)_{m n i j}+\left[\Sigma^{n+1}-2 \mathbf{A}^{\mathbf{e l}_{n}^{n+1}} \Sigma^{n+1}\right]_{m n}\left[\mathscr{H}^{-1} \overline{\mathscr{M}}\right]_{m n i j}
$$

and using the symmetric properties of $\Sigma, \mathbf{A}^{\mathrm{el}}$ and $\mathscr{H}^{-1} \overline{\mathscr{M}}$, relation (A.33) leads to

$$
\left[\frac{\partial \mathbf{A}_{n}^{\mathbf{e}^{n+1}}}{\partial\left[x^{n+1}\right]^{\mu}}: \Sigma^{n+1}\right] \mathbf{A}_{n}^{n+1}=\mathscr{C}^{* * 6} \bullet\left[\vec{B}^{n+1}\right]^{\mu}
$$

with $\mathscr{C}_{i j k l}^{* *^{6}}=\mathbf{A}_{i j} \mathbf{C}_{k l}^{* *^{6}}$. The last term of the expression (A.25), is directly obtained from relation (A.24)

$$
-\left[\frac{\partial \boldsymbol{\Sigma}^{n+1}}{\partial\left[\vec{x}^{n+1}\right]^{\mu}}: \mathbf{A}_{n}^{\mathbf{p}^{n+1}}\right] \mathbf{A}_{n}^{n+1}=\mathscr{C}^{* * 7} \bullet\left[\vec{B}^{n+1}\right]^{\mu}
$$

with $\mathscr{C}_{i j k l}^{* *^{7}}=-\mathbf{A}_{i j} \mathbf{A}_{m n}^{\mathrm{pl}}\left[\mathscr{J}\left(\Sigma^{n+1}\right)+\overline{\mathscr{M}}\right]_{m n k l}$. And finally, using relations (A.26), (A.27), (A.28), (A.29), (A.30), (A.35) and (A.36), tensor $\mathscr{C}^{* *}$ defined in relation (A.25) becomes

$$
\begin{aligned}
\mathscr{C}^{* *}= & {\left[\frac{\frac{D_{\text {int }}}{J_{0}^{n+1}} \mathbf{A}_{n}^{\mathbf{p l}^{n+1}}: \boldsymbol{\Sigma}^{n+1}}{\mathbf{A}_{n}^{n+1}: \mathbf{A}_{n}^{n+1}}\right] \mathscr{C}^{* * 1}+\left[\frac{D_{\text {int }}}{J_{0}^{n+1}}-\mathbf{A}_{n}^{\mathbf{p}^{n+1}}: \Sigma^{n+1}\right] \mathscr{C}^{* * 2}+\left[\frac{1}{J_{0}^{n+1} \mathbf{A}_{n}^{n+1}: \mathbf{A}_{n}^{n+1}}\right] \mathscr{C}^{* * 3} } \\
& +\left[\frac{D_{\text {int }}}{\mathbf{A}_{n}^{n+1}: \mathbf{A}_{n}^{n+1}}\right] \mathscr{C}^{* * 4}+\left[\frac{1}{\mathbf{A}_{n}^{n+1}: \mathbf{A}_{n}^{n+1}}\right] \mathscr{C}^{* * 5}+\left[\frac{1}{\mathbf{A}_{n}^{n+1}: \mathbf{A}_{n}^{n+1}}\right] \mathscr{C}^{* * 6}+\left[\frac{1}{\mathbf{A}_{n}^{n+1}: \mathbf{A}_{n}^{n+1}}\right] \mathscr{C}^{* * 7}
\end{aligned}
$$


Finally, the forth term of relation (A.18) is easily obtained since

$$
\frac{\partial\left[\mathbf{f}_{0}^{n+1^{\mathrm{T}}} D^{\xi} J_{0}^{n+1}\right]_{i}}{\partial\left[\vec{x}^{n+1}\right]_{k}^{\mu}}=\left[\vec{B}^{n+1}\right]_{j}^{\xi}\left[-\delta_{j k} \delta_{i l}+\delta_{i j} \delta_{k l}\right]\left[\vec{B}^{n+1}\right]_{l}^{\mu} J_{0}^{n+1}
$$

Therefore, if we define $\mathscr{G}(\mathbf{M})$, the geometric tensor, such that

$$
\mathscr{G}(\mathbf{M})=-\mathbf{M}_{i l} \delta_{j k}+\mathbf{M}_{i j} \delta_{k l}
$$

and thanks to relations (A.19), (A.24), (A.25) and (A.38) we can rewrite the relation (A.18) as

$$
\mathbf{K}^{* *^{\xi \mu}}=\frac{1}{2} \int_{V_{0}}\left\{\left[\vec{B}^{n+1}\right]^{\xi} \bullet \mathscr{K}^{* *} \bullet\left[\vec{B}^{n+1}\right]^{\mu} J_{0}^{n+1}\right\} \mathrm{d} V_{0}
$$

with

$$
\mathscr{K}_{i j k l}^{* *}=\mathscr{N}_{i j k l}^{2}+\left[\mathbf{f}_{n}^{n+1}+\mathbf{I}\right]_{i m}\left[\overline{\mathscr{M}}+\mathscr{J}\left(\Sigma^{n+1}\right)+\mathscr{C}^{* *}+\mathscr{G}\left(\Sigma^{n+1}+\mathbf{C}^{* *}\right)\right]_{m j k l}
$$

The final expression of the stiffness matrix is obtained from relation (A.17) and from relation (A.40)

$$
\mathbf{K}^{\xi \mu}=\frac{\partial\left[\vec{F}_{\text {int }}\right]^{\xi}}{\partial\left[\vec{x}^{n+1}\right]^{\mu}}=\frac{1}{4} \int_{V_{0}}\left\{\left[\vec{B}^{n}\right]^{\xi} \bullet \mathscr{K}^{*} \bullet\left[\vec{B}^{n}\right]^{\mu} J_{0}^{n}+\left[\vec{B}^{n+1}\right]^{\xi} \bullet \mathscr{K}^{* *} \bullet\left[\vec{B}^{n+1}\right]^{\mu} J_{0}^{n+1}\right\} \mathrm{d} V_{0}
$$

Let us note that this stiffness matrix is not symmetric. Nevertheless, since the relation (A.23) gives a nonsymmetric four order tensor, the use of the Newmark scheme gives also a non-symmetric stiffness matrix.

\section{Appendix B. Material tensors}

Now we have to develop tensor $\overline{\mathscr{M}}$ defined in relation (A.13) and tensor $\mathbf{D}_{\text {int }}$ defined in relation (A.7). The material tensor can be found in Nagtegaal and Veldpaus (1984) and Ponthot (2002)

$$
\overline{\mathscr{M}}_{i j k l}=k \delta_{i j} \delta_{k l}+g^{*}\left[\delta_{i l} \delta_{j k}+\delta_{i k} \delta_{j l}-\frac{2}{3} \delta_{i j} \delta_{k l}-2 \mu^{*} \mathbf{N}_{i j} \mathbf{N}_{k l}\right]
$$

with $g^{*}=\beta g$ and

$$
\begin{aligned}
\beta & =\sqrt{\frac{2}{3}} \frac{\Sigma^{\mathrm{v} n+1}+\bar{\alpha}^{n+1}-\bar{\alpha}^{n}}{\sqrt{\left[\mathbf{s}^{\mathbf{e}}-\boldsymbol{\alpha}\right]:\left[\mathbf{s}^{\mathbf{e}}-\boldsymbol{\alpha}\right]}} \\
\mu^{*} & =\frac{g^{*}}{1+\frac{h}{3 g^{*}+[\beta-1] h}} \\
h & =\frac{\partial \Sigma^{\mathrm{v} n+1}}{\partial \varepsilon^{\mathrm{p}}}
\end{aligned}
$$

Let us note that

$$
\mathscr{H}_{i j m n}^{-1} \overline{\mathscr{M}}_{m n k l}=\frac{1}{3} \delta_{i j} \delta_{k l}+\beta\left[\frac{1}{2} \delta_{i l} \delta_{j k}+\frac{1}{2} \delta_{i k} \delta_{j l}-\frac{1}{3} \delta_{i j} \delta_{k l}-\mu^{*} \mathbf{N}_{i j} \mathbf{N}_{k l}\right]
$$

To obtain tensor $\mathbf{D}_{\text {int }}$, we have to derive relation (73)

$$
\begin{aligned}
\frac{\partial D_{\text {int }}}{\partial\left[\vec{x}^{n+1}\right]^{\mu}}= & \frac{1}{2}\left\{\left[\sum_{\mathrm{v}}^{n+1}+\bar{\alpha}^{n+1}\right] J_{0}^{n+1}+\left[\sum_{\mathrm{v}}^{n}+\bar{\alpha}^{n}\right] J_{0}^{n}\right\} \frac{\partial \varepsilon_{n}^{\mathrm{p} n+1}}{\partial\left[\vec{x}^{n+1}\right]^{\mu}}+\frac{1}{2}\left[h \varepsilon_{n}^{\mathrm{p} n+1}+\bar{\alpha}^{n+1}-\bar{\alpha}^{n}\right] J_{0}^{n+1} \frac{\partial \varepsilon^{\mathrm{p} n+1}}{\partial\left[\vec{x}^{n+1}\right]^{\mu}} \\
& +\frac{1}{2}\left[\sum_{\mathrm{v}}^{n+1}+\bar{\alpha}^{n+1}\right] \varepsilon_{n}^{\mathrm{p}} n+1 \frac{\partial J_{0}^{n+1}}{\partial\left[\vec{x}^{n+1}\right]^{\mu}}
\end{aligned}
$$

with (Nagtegaal and Veldpaus, 1984; Ponthot, 2002) 


$$
\frac{\partial \varepsilon_{n}^{\mathrm{p} n+1}}{\partial\left[\vec{x}^{n+1}\right]^{\mu}}=\sqrt{\frac{2}{3}}\left[\mu^{*}(\beta-1)+1\right] \mathbf{N}\left[\vec{B}^{n+1}\right]^{\mu}
$$

and with

$$
\frac{\partial J_{0}^{n+1}}{\partial\left[\vec{x}^{n+1}\right]^{\mu}}=J_{0}^{n+1} \mathbf{I}\left[\vec{B}^{n+1}\right]^{\mu}
$$

Therefore, it comes

$$
\begin{aligned}
\mathbf{D}_{\text {int }}= & \left\{\left[\sum_{\mathrm{v}}^{n+1}+\bar{\alpha}^{n+1}+\left(h \varepsilon_{n}^{\mathrm{p} n+1}+\bar{\alpha}^{n+1}-\bar{\alpha}^{n}\right)\right] J_{0}^{n+1}+\left[\sum_{\mathrm{v}}^{n}+\bar{\alpha}^{n}\right] J_{0}^{n}\right\} \frac{1}{2} \sqrt{\frac{2}{3}}\left[\mu^{*}(\beta-1)+1\right] \mathbf{N} \\
& +\frac{1}{2}\left[\sum_{\mathrm{v}}^{n+1}+\bar{\alpha}^{n+1}\right] J_{0}^{n+1} \mathbf{I}
\end{aligned}
$$

\section{References}

Adam, L., 2003. Modélisation thermomécanique en grandes déformations de la mise à forme anisotherme des métaux. Application à la superplasticité. Ph.D. Thesis, Université de Liège (in French).

Antman, S., 1995. Nonlinear Problems of Elasticity. Springer-Verlag.

Armero, F., Petöcz, E., 1998. Formulation and analysis of conserving algorithms for frictionless dynamic contact/impact problems. Computer Methods in Applied Mechanics and Engineering 158, 269-300.

Armero, F., Petöcz, E., 1999. A new dissipative time-stepping algorithm for frictional contact problems: formulation and analysis. Computer Methods in Applied Mechanics and Engineering 179, 151-178.

Armero, F., Romero, I., 1999. Dissipative integration algorithms for nonlinear elastodynamics. In: Wunderlich, W. (Ed.), ECCM99, Proceedings of the European Conference on Computational Mechanics, Munich, Germany, CD-ROM.

Armero, F., Romero, I., 2001a. On the formulation of high-frequency dissipative time-stepping algorithms for non-linear dynamics. Part I: low-order methods for two model problems and nonlinear elastodynamics. Computer Methods in Applied Mechanics and Engineering 190, 2603-2649.

Armero, F., Romero, I., 2001b. On the formulation of high-frequency dissipative time-stepping algorithms for non-linear dynamics. Part II: second-order methods. Computer Methods in Applied Mechanics and Engineering 190, 6783-6824.

Bajer, A., Demkowicz, L., 2002. Dynamic contact/impact problems, energy conservation, and planetary gear trains. Computer Methods in Applied Mechanics and Engineering 191, 4159-4191.

Bauchau, O., Bottasso, C., 1999. On the design of energy preserving and decaying schemes for flexible, nonlinear multi-body systems. Computer Methods in Applied Mechanics and Engineering 169, 61-79.

Bauchau, O., Bottasso, C., Trainelli, L., 2003. Robust integration schemes for flexible multibody systems. Computer Methods in Applied Mechanics and Engineering 192, 395-420.

Bauchau, O., Choi, J.-Y., Bottasso, C., 2002. Time integrators for shells in multibody dynamics. Computers \& Structures 80, 871-889.

Bauchau, O., Joo, T., 1999. Computational schemes for non-linear elastodynamics. International Journal of Numerical Methods in Engineering 45, 693-719.

Bauchau, O., Theron, N., 1996. Energy decaying scheme for non-linear beam models. Computer Methods in Applied Mechanics and Engineering 134, 37-56.

Belytschko, T., Hughes, T., 1983. Computational Methods for Transient Analysis. North-Holland.

Belytschko, T., Liu, W., Moran, B., 2000. Nonlinear Finite Elements for Continua and Structures. John Wiley.

Belytschko, T., Schoeberle, D., 1975. On the unconditional stability of an implicit algorithm for non-linear structural dynamics. Journal of Applied Mechanics 42, 865-869.

Betsch, P., Steinmann, P., 2000a. Conservation properties of a time fe method. Part i: Time-stepping schemes for $n$-body problems. International Journal of Numerical Methods in Engineering 49, 599-638.

Betsch, P., Steinmann, P., 2000b. Inherent energy conserving time finite elements for classical mechanics. Journal of Computational Physics 160, 88-116.

Betsch, P., Steinmann, P., 2001. Conservation properties of a time fe method. Part ii: Time-stepping schemes for non-linear elastodynamics. International Journal of Numerical Methods in Engineering 50, 1931-1955.

Borri, M., Bottasso, L., Trainelli, L., 2001. Integration of elastic multibody system by invariant conserving/dissipating algorithms. i. formulation. Computer Methods in Applied Mechanics and Engineering 190, 3669-3699. 
Bottasso, C., Bauchau, O., Choi, J.-Y., 2002. An energy decaying scheme for non-linear dynamics of shells. Computer Methods in Applied Mechanics and Engineering 191, 3099-3121.

Bottasso, C., Borri, M., 1997. Energy preserving/decaying schemes for non-linear beam dynamics using the helicoidal approximation. Computer Methods in Applied Mechanics and Engineering 143, 393-415.

Bottasso, C., Borri, M., 1998. Integrating finite rotation. Computer Methods in Applied Mechanics and Engineering 164, 307-331.

Bottasso, L., Borri, M., Trainelli, L., 2001. Integration of elastic multibody system by invariant conserving/dissipating algorithms. ii. numerical schemes and applications. Computer Methods in Applied Mechanics and Engineering 190, 3701-3733.

Brank, B., 2002. An energy conserving non-linear dynamic finite formulation for flexible composite laminates. Computers \& Structures 80, 677-689.

Briseghella, L., Majorana, C., Pellegrino, C., 1999. Conservation of angular momentum and energy in the integration of non-linear dynamics equations. Computer Methods in Applied Mechanics and Engineering 179, 247-263.

Chawla, V., Laursen, T., 1998. Energy consistent algorithms for frictional contact problems. International Journal of Numerical Methods in Engineering 42, 799-827.

Chung, J., Hulbert, G., 1993. A time integration algorithms for structural dynamics with improved numerical dissipations: the generalized- $\alpha$ method. Journal of Applied Mechanics 60, 371-375.

Demkowicz, L., Bajer, A., 2001. Conservative discretization of contact/impact problems for nearly rigid bodies. Computer Methods in Applied Mechanics and Engineering 190, 1903-1924.

Goicolea, J., Garcia Orden, J., 2000. Dynamic analysis of rigid and deformable multibody systems with penalty methods and energymomentum schemes. Computer Methods in Applied Mechanics and Engineering 188, 789-804.

Gonzalez, O., 2000. Exact energy and momentum conserving algorithms for general models in nonlinear elasticity. Computer Methods in Applied Mechanics and Engineering 190, 1763-1783.

Gonzalez, O., Simo, J., 1996. On the stability of sympletic and energy-momentum algorithms for non-linear hamiltonian systems with symmetry. Computer Methods in Applied Mechanics and Engineering 134, 197-222.

Géradin, M., Cardona, A., 2000. Flexible Multibody Dynamics (A Finite Element Approach). John Wiley.

Géradin, M., Rixen, D., 1994. Mechanical Vibrations (Theory and Applications to Structural Dynamics). John Wiley, Paris.

Graham, E., Jelenić, G., Crisfield, M., 2002. A note on the equivalence of two recent time-integration schemes for $n$-body problems. Communication in Numerical Method in Engeneering 18, 615-620.

Hughes, T., 1977. A note on the stability of newmark's algorithm in nonlinear structural dynamics. International Journal of Numerical Methods in Engineering 11 (2), 383-386.

Hughes, T., 1987. The Finite Element Method. Prentice-Hall.

Hughes, T., Caughey, T., Liu, W., 1978. Finite-element method for nonlinear elastodynamics which conserve energy. Journal of Applied Mechanics 45 (June), 366-370.

Ibrahimbegovic, A., Mamouri, S., 2002. Energy conserving/decaying implicit time-stepping scheme for non-linear dynamics of threedimensional beams undergoing finite rotations. Computer Methods in Applied Mechanics and Engineering 191, 4241-4258.

Kane, C., Mardsen, J., Ortiz, M., West, M., 2000. Variational integrators and the newmark algorithm for conservative and dissipative mechanical system. International Journal of Numerical Methods in Engineering 49, 1295-1325.

Kuhl, D., Crisfield, M., 1999. Energy-conserving and decaying algorithms in non-linear structural dynamics. International Journal of Numerical Methods in Engineering 45, 569-599.

Kuhl, D., Ramm, E., 1996. Constraint energy momentum algorithm and its application to non-linear dynamics of shells. Computer Methods in Applied Mechanics and Engineering 136, 293-315.

Kuhl, D., Ramm, E., 1999. Generalized energy-momentum method for nonlinear adaptive shell dynamics. Computer Methods in Applied Mechanics and Engineering 178, 343-366.

Laursen, T., 2002. Computational Contact and Impact Mechanics. Springer.

Laursen, T., Chawla, V., 1997. Design of energy conserving algorithms for frictionless dynamic contact problems. International Journal of Numerical Methods in Engineering 40, 863-886.

Laursen, T., Love, G., 2002. Improved implicit integrators for transient impact problems-geometric admissibility within the conserving framework. International Journal of Numerical Methods in Engineering 53, 245-274.

Laursen, T., Meng, X., 2001. A new solution procedure for application of energy-conserving algorithms to general constitutive models in nonlinear elastodynamics. Computer Methods in Applied Mechanics and Engineering 190, 6309-6322.

Lubliner, J., 1990. Plasticity Theory. Macmillan.

Maenchen, M., Sack, S., 1964. The tensor code. Methods of Computational Physics 3, 387-400.

Matthies, H., Strang, G., 1979. The solution of nonlinear finite element equations. International Journal of Numerical Methods in Engineering 14, 1613-1626.

Meng, X., Laursen, T., 2001. Energy consistent algorithms for dynamic finite deformation plasticity. Computer Methods in Applied Mechanics and Engineering 191, 1639-1675.

Meng, X., Laursen, T., 2002. On energy consistency of large deformation plasticity models, with application to the design of unconditionally stable time integrators. Finite Elements in Analysis and Design 38, 949-963. 
Miehe, C., Schröder, J., 2001. Energy and momentum conserving elastodynamics of a non-linear brick-type mixed finite shell element. International Journal of Numerical Methods in Engineering 50, 1801-1823.

Nagtegaal, J., 1982. On the implementation of inelastic constitutive equations with special reference to large deformation problems. Computer Methods in Applied Mechanics and Engineering 33, 469-484.

Nagtegaal, J., Veldpaus, F., 1982. Analysis of metal forming problems with an improved finite strain plasticity formulation, in: Numerical Methods in Industrial Forming Processes. Pineridge, Swansea, UK.

Nagtegaal, J., Veldpaus, F., 1984. On the implementation of finite strain plasticity equations in a numerical model, in: Pittman, et al. (Eds.), Numerical Analysis of Forming Processes. John Wiley.

Newmark, N., 1959. A method of computation for structural dynamics. Journal of the Engineering Mechanics Division ASCE 85 (EM3), 67-94.

Noels, L., Stainier, L., Ponthot, J., 2004. Energy-momentum conserving algorithm for non-linear hypoelastic constitutive models. International Journal of Numerical Methods in Engineering, 59.

Ponthot, J., 2002. Unified stress update algorithms for the numerical simulation of large deformation elasto-plastic and elastoviscoplastic processes. International Journal of Plasticity 18, 91-126.

Ponthot, J.-P., 1995. Traîtement unifié de la mécanique des milieux continus solides en grandes transformations par la méthode des éléments finis. Ph.D. thesis, Université de Liège (in French).

Romero, I., Armero, F., 2000. High-frequency dissipative time-stepping algorithms for the dynamics of nonlinear shells. In: Onate, E., Brigeda, G., Swález, B. (Eds.), ECCOMAS 2000, Proceedings of the Congress on Computational Methods in Applied Sciences and Engineering, Barcelona, Spain.

Romero, I., Armero, F., 2002a. Numerical integration of the stiff dynamics of geometrically exact shells: an energy-dissipative momentum-conserving scheme. International Journal of Numerical Methods in Engineering 54, 1043-1086.

Romero, I., Armero, F., 2002b. An objective finite element approximation of the kinematics of geometrically exact rods ans its use in the formulation of an energy-momentum conserving scheme in dynamics. International Journal of Numerical Methods in Engineering 54, 1683-1716.

Sansour, C., Wagner, W., Wriggers, P., Sansour, J., 2002. An energy-momentum integration scheme and enhanced strain finite elements for nonlinear dynamics of shells. International Journal of Nonlinear Mechanics 37, 951-966.

Simo, J., Gonzalez, O., 1994. Recent results on the numerical integration of infinite-dimensional hamiltonian system, in: Hughes, T., Oñate, E., Zienkiewicz, O. (Eds.), Recent Developments in Finite Element Analysis. CIMNE, Barcelona, Spain, pp. $255-271$.

Simo, J., Hughes, T., 1998. Computational Inelasticity. Springer.

Simo, J., Tarnow, N., 1992. The discrete energy-momentum method. conserving algorithms for nonlinear elastodynamics. ZAMP 43, $757-792$.

Simo, J., Tarnow, N., 1994. A new energy and momentum conserving algorithms for the nonlinear dynamics of shells. International Journal of Numerical Methods in Engineering 37, 2527-2549.

Simo, J., Tarnow, N., Wong, K., 1992. Exact energy-momentum conserving algorithms and sympletic schemes for nonlinear dynamics. Computer Methods in Applied Mechanics and Engineering 100, 63-116.

Wilkins, M., 1964. Calculation of elastoplastic flows. Methods of Computational Physics 3, 211-263.

Zhong, H., Crisfield, M., 1998. An energy-conserving co-rotational procedure for the dynamics of shell structures. Engineering Computations 15 (5), 552-576. 